\title{
Membrane-Bound O-Acyltransferase 7 (MBOAT7) is a Key Regulator of Glycolysis in Clear Cell Renal Carcinoma.
}

Chase K. A. Neumann ${ }^{1,2}$, William Massey ${ }^{1,2}$, Danny Orabi ${ }^{1,2}$, Renliang Zhang ${ }^{1}$, Daniel J. Silver $^{1}$, Justin D. Lathia ${ }^{1,2,3,4}$, J. Mark Brown ${ }^{1,2,4,5^{*}}$

1. Department of Cardiovascular and Metabolic Sciences, Lerner Research Institute, Cleveland Clinic, Cleveland, Ohio, 44195, USA

2. Department of Molecular Medicine, Cleveland Clinic Lerner College of Medicine of Case Western Reserve University, Cleveland, Ohio, 44195, USA

3. Rose Ella Burkhardt Brain Tumor and Neuro-Oncology Center, Cleveland Clinic, Cleveland, Ohio, 44195, USA.

4. Comprehensive Cancer Center, Case Western Reserve University, Cleveland, Ohio, 44106, USA.

5. Center for Microbiome and Human Health, Cleveland Clinic Foundation, Cleveland, Ohio, 44195, USA

* = To whom correspondence should be addressed: Department of Cardiovascular \& Metabolic Sciences, Cleveland Clinic, Cleveland, OH 44195, USA. Tel: 216-444-8340; Fax: 216-444-9404; E-mail: brownm5@ccf.org

Keywords: cancer, metabolism, lipid, phosphatidylinositol, glycolysis 


\section{Abstract}

Objective: The most common and deadliest urological cancer is clear cell Renal Cell Carcinoma (ccRCC). ccRCC is characterized by striking reorganization of both carbohydrate and lipid metabolism. It was recently demonstrated that lipid remodeling enzyme Membrane-Bound O-Acyltransferase 7 (MBOAT7) that generates phosphatidylinositol (PI) is important for the ccRCC progression. However, whether MBOAT7-driven PI remodeling is associated with other metabolic alterations commonly found in cCRCC is poorly understood.

Methods: MBOAT7 deficient cCRCC cell lines were generated by genome editing, and were characterized by a general reduction in glycolytic capactiy. Using targeted metabolomics approach in Caki-1 cells, we measured the glycolytic intermediates and the relative expression of key glycolytic enzymes. We also measured basal respiration and maximal respiration with MBOAT7 deficiency in the presence of glucose. Lastly, in vivo xenograft studies were performed with parental and MBOAT7 deficient cells.

Results: MBOAT7 deficiency was associated with a reduction in glycolytic gene expression and protein abundance. In parallel, we found that glycolytic intermediates similarly decreased which may contribute to a reduction in glycolysis. MBOAT7 deficiency reduces basal respiration and maximal respiration. Similarly, we see maximum glycolytic capacity also reduced with MBOAT7 loss of function. Finally, the in vivo xenograft demonstrated MBOAT7 knockout significantly increased overall survival and reduced glycolytic HK2 protein abundance in vivo.

Conclusions: Our work highlights MBOAT7 as a key regulator of glycolysis in ccRCC. Our data provides additional evidence that suggests MBOAT7 as a novel target to regulate tumor growth in vivo. 


\section{Introduction}

The most prevalent and devastating kidney cancer is clear cell Renal cell carcinoma (ccRCC) [1]. ccRCC has a large lipid rich tumor component compared to other solid tumors [2-4]. Several studies have demonstrated a lipid droplet protein (PLIN2) is a biomarker of ccRCC tumors [4,5]. Past studies in other models have shown, signaling phospholipids such as phosphatidylinositol and phosphoinositides (PIPs) are critical for life $[6,7]$. Previous work has demonstrated that $\mathrm{PI} 3 \mathrm{~K}$ and $\mathrm{PIP}_{3,4,5}$ is critical for Aldolase mobilization to facilitate glycolysis [8]. This work demonstrates a clear link between phosphoinositide signaling and glycolytic regulation.

Similar to many other cancers, ccRCC is characterized by high glycolytic rates that support Warburg metabolism [9]. With dependency on glycolysis, ccRCC upregulates glucose transporter GLUT1, increases the rate-limiting hexokinase II (HK2), and several other members of glycolysis through hypoxia inducible factors (HIF). Although it is appreciated that glucose metabolism is altered in ccRCC renal physiology upon transformation, the mechanisms of modulating glucose metabolism following transformation is less understood [10].

Furthermore, our recent work demonstrated arachidonic acid-containing phosphatidylinositol (AA-PI) and Lands' Cycle remodeling enzyme, MBOAT7, is important for tumor PI generation, downstream signaling and tumor formation (Neumann et al. 2020. Mol. Metab. In Press). However, the mechanism by which MBOAT7 altered tumor biology was not completely understood. MBOAT7 alters specifically $\mathrm{PI}$ and is not promiscuous across other phospholipid species [11,12]. MBOAT7 knockout has been shown to decrease phosphoinositide levels in multiple tissues through decreasing substrate availability $[12,13]$. A key ccRCC cell line used in this study is $786-0$; this line has a known PTEN mutation which confers a more aggressive and metabolically active 
phenotype in vivo and in vitro $[14,15]$. Although lipid metabolism and ccRCC has recently been studied [16], little has been done to address the relationship between lipid metabolism and glycolysis. Here we demonstrate that MBOAT7 deficiency decreases glycolysis through Aldolase and HK2. MBOAT7 loss of function reduces mitochondrial respiration and ATP production, which results in a dramatic increased survival in vivo in the 786-O xenograft model. These data support the hypothesis that limiting phosphoinositide phospholipids reduces glycolysis and may hold therapeutic promise. 


\section{Methods}

2.1 Cell Lines and Cell Culture Conditions. Caki-1 and 786-O cell lines were obtained from American Type Culture Collection (ATCC), and were confirmed to be mycoplasma free. These cells were maintained in McCoy's 5A Medium with 10\% FBS and 1\% Pen/Strep. Confirmed MBOAT7 deficient clones were utilized from previous studies (Neumann et al. 2020. Mol. Metab. In Press). Caki-1 and 786-O expansions were propagated no longer than eighteen consecutive passages.

2.2 RNA isolation, quantitative real time-PCR, and RNAseq dataset. Total RNA was isolated the RNeasy isolation method using manufacturer's recommendations (Qiagen and Thermo Fisher). RNA concentrations were quantified using a Nanodrop 2000. mRNA expression levels were calculated based on the $\Delta \Delta-C T$ method. Quantitative real timePCR (qRT-PCR) was conducted using the Applied Biosystems 7500 Real-Time PCR System. Primers used for qRT-PCR are available in Table 1. We used the RNAseq dataset previously published from the Caki-1 cell line available with the GEO accession: GSE131881 (Neumann et al. 2020. Mol. Metab. In Press).

2.3 Western Blot Analysis. Cell tissue lysates were generated using a modified RIPA buffer and western blotting was performed following the previously described methods [17]. Antibodies used are available through Sigma Aldrich or Cell Signaling with the corresponding product numbers in Table 2.

2.4 Seahorse Metabolic Fitness Studies. Following the Agilent Seahorse manufacturers recommended protocol, we plated cell populations into XFe24 culture plates the day before assay at 50,000 cells per well in normal growth media (McCoy's 5A). Both Caki-1 and 786-O were used at the same cell density. Mitochondrial Stress Test: The media was changed from growth media to assay media 1 hour prior to testing. DMEM Agilent base media $+10 \mathrm{mM}$ glucose for the mitochondrial stress test was used 
for the initial assays. The traditional Mito Stress Test assay was used with all studies Oligomycin 1uM (Port A), FCCP 1uM (Port B), and Rot/AA 0.5 uM (Port C). Glycolysis Stress Test: The media was changed from growth media to base media or base media + $1 \mathrm{mM}$ pyruvate at 1 hour prior to testing. Following manufacturers recommendations Glucose 10mM (Port A), Oligomycin 1uM (Port B), and 2-DG 50mM (Port C). Both Glycolysis and Mitochondrial Stress Test were normalized to well protein concentrations to account for variability in cell number.

2.5 In Vivo ccRCC Xenograft Studies. MBOAT7 ${ }^{+/+}$and MBOAT7 $7^{-/-} 786-\mathrm{O}$ cell lines were injected in the subcutaneous flank of NSG animals (Jackson laboratory) at 2.5 million cells per mouse in PBS ( $n=10$ per group). Once tumors were palpable, digital caliper measurements were used to follow tumor growth over time. Endpoint criteria were defined as $15 \mathrm{~mm}$ tumor width or length, these animals were then necropsied and reached endpoint. All mice studies were approved by the Institutional Animal Care and Use Committee of the Cleveland Clinic.

2.6 Statistical Analysis. All figures are shown with \pm SEM. For comparisons of three groups, we utilized one-way ANOVA with a post hoc Tukey test. Log-rank test (MantelCox test) was used to compare survival differences between two groups of patients. When comparing two groups, we used an unpaired Student $t$ test or multiple $t$ test. The $P$ values significance cutoffs for all tests used are as follows: $p$-value $<0.05\left({ }^{*}\right), 0.002\left({ }^{* *}\right)$, $0.0002\left(^{* * *}\right),<0.0001\left(^{* * * *}\right)$. 


\section{Results}

Loss of MBOAT7 correlates with a decrease in glucose consumption and glycolytic gene expression. To study the role of MBOAT7 and PI metabolism on glycolysis, we utilized previously published MBOAT7 deficient cCRCC Caki-1 and 786-O cell. We observed that with MBOAT7 loss of function, the acidification of media occurs more slowly compared to wild-type (Figure 1A). Utilizing a previously described RNAseq dataset in the Caki-1 cell line (GSE131881), we see that MBOAT7 deficiency leads to a significant reduction in the glycolytic gene set after gene set enrichment analysis (GSEA) (Figure 1B). Looking specifically at the initial steps of glycolysis, there is a reduction in the hexokinase 2 (HK2), glucose-6-phosphate isomerase (GPI), and phosphofructokinase (PFKP) with MBOAT7 loss of function in the RNAseq dataset (Figure 1C). In vitro experiments, we see that MBOAT7 deficiency leads to a decrease in glucose consumption and lactate production (Figure 1D), consistent with decreased glycolysis.

MBOAT7 deficiency reduces pyruvate and several key glycolytic enzymes in HK2 and $A L D O C$. We pursued targeted mass spectrometry for the glycolytic intermediates in the Caki-1 model following high glucose supplementation for 1 hour. Dihydroxyacetone and pyruvate, an intermediate and product of glycolysis (Figure 2A) demonstrates a significant reduction following MBOAT7 loss of function, which corresponds with the loss of gene expression in HK2 and ALDOC (Figure 2B). To validate the reduction in HK2 expression, we performed western blotting and see that HK2 protein abundance decreases with MBOAT7 deficiency in the Caki-1 and 786-O models (Figure 2C). These data suggest alterations in glycolysis and metabolic fitness with MBOAT7 deficiency.

MBOAT7 loss of function reduces glycolysis and metabolic fitness. To assess glucose metabolism, we utilized the Seahorse Metabolic Stress Tests. Oxygen consumption rate $(\mathrm{OCR})$ is directly related to ATP production through the electron 
transport chain. In our initial tests with glucose, MBOAT7 deficient Caki-1 cells have a reduced basal and maximal respiration (Figure 3A). Our findings are validated in the 786-O RCC model that basal and maximal respiration are reduced with the loss of MBOAT7 (Figure 3B). MBOAT7 deficiency significantly reduces ATP production in the Caki-1 and 786-O cell models (Figure 3C,D). To assess glucose utilization, we performed glycolysis stress test in the presence of base media alone, which demonstrated a reduction in maximum glycolysis with MBOAT7 deficiency (Figure 3E). This significant reduction in glycolysis could be rescued with pyruvate supplementation into the media (Figure 3F).

MBOAT7 loss of function decreases in vivo tumor volume and glycolysis. To test the overall tumor growth, MBOAT7 wild type or deficient 786-O cells were injected into the flanks of Nod SCID Gamma (NSG) mice. In this experiment, we see an increased in vivo overall survival with MBOAT7 loss of function (Figure 4A). Accordingly, the tumor volume at end-point for all animals is substantially reduced with MBOAT7 deficiency (Figure 4B). To confirm these data, we utilized histology and immunohistochemistry to stain for the H\&E (architecture), Ki67 (cell division), and Hexokinase 2 (glycolysis) (Figure 4C). The quantification of tumor staining with or without MBOAT7 demonstrates a reduction in $\mathrm{Ki} 67^{+}$staining per field and trending reductions in HK2 staining (Figure 4D). The in vivo gene expression shows a significant reduction in the rate-limiting glycolytic pathway member, HK2, within MBOAT7 deficient tumors (Figure 4E). Other metabolic regulators were assessed in these tumors such as CPT1A, PPARA, and PPARG (Supplementary). Additionally, we also see trending increases in Trichrome fibrosis staining in the MBOAT7 wild-type tumors (Supplementary). Mechanistically, MBOAT7 deficiency leads to a decrease in in vivo P-S6K and P-ERK signaling, while maintaining reduced HK2 protein abundance (Figure 4F). 


\section{Discussion}

A hallmark of many cancers including kidney cancer is the unrelenting metabolic adaptation and glycolysis that occurs. To meet metabolic demand, tumor metabolism increases glycolysis, which provides a relatively inefficient ATP production. The Warburg effect in full force leads to increased glycolysis and substrate influx to help fuel the increased anabolism for cell division and proliferation. Glycolytic gene expression can be altered through multiple mechanisms including HIF and mTORC [18-21], the continued signaling through these nodes can lead to increases in glycolytic enzymatic intermediates. Others have shown PI3K and subsequent product $\left(\mathrm{PIP}_{3,4,5}\right)$ is critical for the downstream signaling and increased glycolytic activity through mTORC1/S6K $[8,15]$.

In our previous study that identified MBOAT7's potential role in cancer, we demonstrated a loss of PI confers a decreased S6K signaling (Neumann et al. 2020. Mol. Metab. In Press). To follow, we see a reduction in glycolysis with MBOAT7 loss of function in both ccRCC cell lines. In this study, we demonstrate in ccRCC that with the loss of a Lands' Cycle remodeling enzyme, MBOAT7, and the reduction in PI leads to reduced glycolysis. The reduced glycolytic gene expression with MBOAT7 deficiency was validated using qRT-PCR, which showed the most striking reductions in glycolytic gene expression presented in the Hexokinase-2 (HK2) and Aldolase (ALDOC) transcripts (Figure 2). Pairing gene expression with the glycolytic intermediate profiles, suggests a reduction in glycolysis is occurring as the product of glycolysis, pyruvate, is reduced by approximately $50 \%$.

Consistent with the reduction in glycolysis, the metabolic fitness with MBOAT7 loss of function suggests a reduction in basal respiration and maximal respiration in the presence of only glucose. MBOAT7 deficient cCRCC lines seem to be not utilizing glucose as efficiently compared to wild-type cells given the same constraints. The 
utilization of glucose for tumor growth and development is incredibly important, and led us to our final experiment.

Previous MBOAT7 loss of function studies utilized the Caki-1 ccRCC cell xenograft, and MBOAT7 deficient xenografts never developed palpable tumors after 9 weeks. In this study, our in vivo experiments utilized a more aggressive 786-O model with a PTEN mutation, and as the mechanism for PTEN enriches in $\mathrm{PIP}_{3}$ species. These MBOAT7 deficient xenografts still developed palpable tumors as we hypothesized due to a more aggressive genomic background, however these tumors are delayed with a slower tumor growth and never reach endpoint criteria. At endpoint MBOAT7 deficient tumors were $\sim 10 \%$ tumor volume of wildtype $786-0$ xenografts.

Interestingly, previous studies have shown that PTEN mutations increase basal glycolysis and cell growth of mouse embryonic fibroblast (MEF) [22]. For the first time, this study demonstrates that limiting upstream PI production through MBOAT7 can limit glycolysis, metabolic fitness, and in vivo tumor growth regardless of PTEN status. This study gives more evidence that PI metabolism helps regulate glycolysis. This also provides rationale for several future studies including assessing MBOAT7/AA-PI role in other PI3K/mTORC1 dependent cancers, in vivo MBOAT7 knockdown interventional study with $P T E N^{\text {mut }} / \mathrm{PI} 3 \mathrm{~K} / \mathrm{mTORC} 1$ active cancer models, developing small molecule inhibitors towards MBOAT7, and determining the role for Lands' Cycle in cancer progression. 


\section{Author Contributions}

C.K.A.N., J.D.L., J.M.B. planned the project, designed experiments, and wrote the manuscript; C.K.A.N., R.Z., D.J.S., W.M., and D.O. executed experiments. C.K.A.N., R.Z., D.J.S., W.M. and D.O. analyzed the data; J.D.L., and J.M.B. provided financial support; all authors were involved in the editing of the final manuscript. 


\section{Acknowledgments}

This study was supported in part by grants provided by the National Institutes of Health (NIH): R01 DK120679 (J.M.B.), P50 AA024333 (J.M.B.), and P01 HL147823 (J.M.B.).

Development of glycolytic mass spectrometry methods reported here were supported by generous pilot grants from the Clinical and Translational Science Collaborative of Cleveland (4UL1TR000439) from the National Center for Advancing Translational Sciences component of $\mathrm{NIH}$ and the NIH Roadmap for Medical Research, the Case Comprehensive Cancer Center (P30 CA043703), the VeloSano Foundation, and a Cleveland Clinic Research Center of Excellence Award. 


\section{Bibliography}

[1] Cohen, H.T., McGovern, F.J., 2005. Renal-Cell Carcinoma. New England Journal of Medicine 353(23): 2477-90, Doi: 10.1056/NEJMra043172.

[2] Gebhard, R.L., Clayman, R.V., Prigge, W.F., Figenshau, R., Staley, N.A., Reesey, C., et al., 1987. Abnormal cholesterol metabolism in renal clear cell carcinoma. Journal of Lipid Research 28(10): 1177-84.

[3] Ackerman, D., Tumanov, S., Qiu, B., Michalopoulou, E., Spata, M., Azzam, A., et al., 2018. Triglycerides Promote Lipid Homeostasis during Hypoxic Stress by Balancing Fatty Acid Saturation. Cell Reports 24(10): 2596-2605.e5, Doi: 10.1016/j.celrep.2018.08.015.

[4] Qiu, B., Ackerman, D., Sanchez, D.J., Li, B., Ochocki, J.D., Grazioli, A., et al., 2015. HIF2a-Dependent Lipid Storage Promotes Endoplasmic Reticulum Homeostasis in Clear-Cell Renal Cell Carcinoma. Cancer Discovery 5(6): 652-67, Doi: 10.1158/2159-8290.CD-14-1507.

[5] Morrissey, J.J., Mellnick, V.M., Luo, J., Siegel, M.J., Figenshau, R.S., Bhayani, S., et al., 2015. Evaluation of Urine Aquaporin-1 and Perilipin-2 Concentrations as Biomarkers to Screen for Renal Cell Carcinoma: A Prospective Cohort Study. JAMA Oncology 1(2): 204-12, Doi: 10.1001/jamaoncol.2015.0213.

[6] Di Paolo, G., De Camilli, P., 2006. Phosphoinositides in cell regulation and membrane dynamics. Nature 443(7112): 651-7, Doi: 10.1038/nature05185.

[7] Janetopoulos, C., Borleis, J., Vazquez, F., lijima, M., Devreotes, P., 2005. Temporal and Spatial Regulation of Phosphoinositide Signaling Mediates Cytokinesis. Developmental Cell 8(4): 467-77, Doi: 10.1016/j.devcel.2005.02.010.

[8] Hu, H., Juvekar, A., Lyssiotis, C.A., Lien, E.C., Albeck, J.G., Oh, D., et al., 2016. Phosphoinositide 3-Kinase Regulates Glycolysis through Mobilization of Aldolase from the Actin Cytoskeleton. Cell 164(3): 433-46, Doi: 10.1016/j.cell.2015.12.042.

[9] Sanderson, S.M., Locasale, J.W., 2018. Revisiting the Warburg Effect: Some Tumors Hold Their Breath. Cell Metabolism 28(5): 669-70, Doi: 10.1016/j.cmet.2018.10.011.

[10] Bailey, S.T., Smith, A.M., Kardos, J., Wobker, S.E., Wilson, H.L., Krishnan, B., et al., 2017. MYC activation cooperates with Vhl and Ink4a/Arf loss to induce clear cell renal cell carcinoma. Nature Communications 8: 15770, Doi: 10.1038/ncomms15770.

[11] Gijón, M.A., Riekhof, W.R., Zarini, S., Murphy, R.C., Voelker, D.R., 2008. Lysophospholipid acyltransferases and arachidonate recycling in human neutrophils. The Journal of Biological Chemistry 283(44): 30235-45, Doi: 10.1074/jbc.M806194200.

[12] Lee, H.-C., Inoue, T., Sasaki, J., Kubo, T., Matsuda, S., Nakasaki, Y., et al., 2012. LPIAT1 regulates arachidonic acid content in phosphatidylinositol and is required for cortical lamination in mice. Molecular Biology of the Cell 23(24): 4689-700, Doi: 10.1091/mbc.e12-09-0673.

[13] Anderson, K.E., Kielkowska, A., Durrant, T.N., Juvin, V., Clark, J., Stephens, L.R., et al., 2013. Lysophosphatidylinositol-Acyltransferase-1 (LPIAT1) Is Required to Maintain Physiological Levels of Ptdlns and PtdInsP2 in the Mouse. PLOS ONE 8(3): e58425, Doi: 10.1371/journal.pone.0058425.

[14] Ramaswamy, S., Nakamura, N., Vazquez, F., Batt, D.B., Perera, S., Roberts, T.M., et al., 1999. Regulation of G1 progression by the PTEN tumor suppressor protein is linked to inhibition of the phosphatidylinositol 3-kinase/Akt pathway. Proceedings of the National Academy of Sciences 96(5): 2110-5, Doi: 10.1073/pnas.96.5.2110. 
[15] Bunney, T.D., Katan, M., 2010. Phosphoinositide signalling in cancer: beyond PI3K and PTEN. Nature Reviews Cancer 10(5): 342-52, Doi: 10.1038/nrc2842.

[16] Du, W., Zhang, L., Brett-Morris, A., Aguila, B., Kerner, J., Hoppel, C.L., et al., 2017. HIF drives lipid deposition and cancer in ccRCC via repression of fatty acid metabolism. Nature Communications 8(1): 1769, Doi: 10.1038/s41467-017-019658.

[17] Helsley, R.N., Varadharajan, V., Brown, A.L., Gromovsky, A.D., Schugar, R.C., Ramachandiran, I., et al., 2019. Obesity-linked suppression of membrane-bound Oacyltransferase 7 (MBOAT7) drives non-alcoholic fatty liver disease. ELife 8: e49882, Doi: 10.7554/eLife.49882.

[18] Semenza, G.L., Roth, P.H., Fang, H.M., Wang, G.L., 1994. Transcriptional regulation of genes encoding glycolytic enzymes by hypoxia-inducible factor 1. Journal of Biological Chemistry 269(38): 23757-63.

[19] Lu, H., Forbes, R.A., Verma, A., 2002. Hypoxia-inducible Factor 1 Activation by Aerobic Glycolysis Implicates the Warburg Effect in Carcinogenesis. Journal of Biological Chemistry 277(26): 23111-5, Doi: 10.1074/jbc.M202487200.

[20] Pusapati, R.V., Daemen, A., Wilson, C., Sandoval, W., Gao, M., Haley, B., et al., 2016. mTORC1-Dependent Metabolic Reprogramming Underlies Escape from Glycolysis Addiction in Cancer Cells. Cancer Cell 29(4): 548-62, Doi: 10.1016/j.ccell.2016.02.018.

[21] Ben-Sahra, I., Manning, B.D., 2017. mTORC1 signaling and the metabolic control of cell growth. Current Opinion in Cell Biology 45: 72-82, Doi: 10.1016/j.ceb.2017.02.012.

[22] N.d. Hexokinase 2-Mediated Warburg Effect Is Required for PTEN and p53Deficiency Driven Prostate Cancer Growth. https://www.ncbi.nlm.nih.gov/pmc/articles/PMC4360961/. [accessed January 29, 2020]. 


\section{FIGURE LEGENDS:}

Figure 1. MBOAT7 deficiency correlates with a reduction in glycolysis A) Conditioned media from cells seeded at the same original density and after 48hrs of culture. B) Gene Set Enrichment Analysis (GSEA) of RNAseq dataset demonstrates a reduction in glycolysis enrichment with MBOAT7 loss of function. C) The first three steps of glycolysis demonstrate a significant reduction with MBOAT7 deficiency. D) Using the supernatant of from the MBOAT7 WT and KO cell lines following 48hrs of media, glucose consumption measurements decrease with loss of function ( $n=4$ per group). Similarly, lactate production measurements after $48 \mathrm{hrs}$ decrease with MBOAT7 deficiency $(\mathrm{n}=4$ per group). Student t-test: ${ }^{*}<0.05,{ }^{* *}<0.0021,{ }^{* * *}<0.0002$

Figure 2. MBOAT7 deficiency decreases glycolytic product, pyruvate. A) Quantitative Real Time PCR (qRT-PCR) shows reduced glycolytic gene expression specifically, 1. HK2 and 4. ALDOC. ( $\mathrm{n}=5$ per group) B) Targeted mass spectrometry of three glycolytic intermediates reveals a reduction in pyruvate with MBOAT7 deficiency. ( $n=4$ per group) C) Western blot analysis of HK1, HK2, and B-Actin in ccRCC cell lines, Caki-1 and 786-O with or without MBOAT7 deficiency. Student t-test: ${ }^{*}<0.05,{ }^{* *}<0.0021,{ }^{* * *}<0.0002$

Figure 3: Metabolic fitness and glucose utilization decreases with the loss of MBOAT7. A) Mitochondrial stress test with Caki-1 WT, KO1, and KO2 in the presence of glucose. B) Mitochondrial stress test with 786-O WT, KO1, and KO2 in the presence of glucose. C) Basal respiration and ATP production during mitochondrial stress test in Caki$1 \mathrm{WT}, \mathrm{KO} 1$, and KO2. D) Basal respiration and ATP production during mitochondrial stress test in 786-O WT, KO1, and KO2. E) Glycolytic Stress Test in the presence of only glucose with Caki-1 cell lines. F) Glycolytic Stress Test in the presence of pyruvate and glucose with Caki-1 cell lines rescues maximal glycolytic capacity. ( $n=5$ per group) $(2=$ independent replicate experiments) Student t-test: ${ }^{*}<0.05,{ }^{* *}<0.0021,{ }^{* * *}<0.0002$ 
Figure 4: Decreased metabolic fitness and MBOAT7 deficiency leads to increased survival in vivo. A) $786-0$ xenografts in NSG female mice show a striking survival advantage with MBOAT7 deficiency. B) Tumor volume of 786-O xenografts after meeting experimental end-point criteria or 70 days post injection. C) Histology representative images of 786-O WT and KO including H\&E, Ki67, and HK2. D) Quantification of Ki67 staining and $\mathrm{HK}^{+}$staining per field in $786-\mathrm{O}$ xenografts. ( $\mathrm{n}=9$ or 7 per group) E) Tumor mRNA expression of MBOAT7 and HK2. ( $n=5$ per group) F) Western blotting analysis of tumor signaling (S6 Kinase and ERK1/2 signaling) and key glycolysis markers (HK1 and HK2). ( $\mathrm{n}=3$ per group) 
Table 1. Quantitative Real Time - PCR Primer Sequences

\begin{tabular}{|c|c|}
\hline Oligo Name & Sequence \\
\hline ENO1_FWD & AATGATAAGACTCGCTATATG \\
\hline ENO1_REV & CGTTCAGTTTCTTGCTAA \\
\hline GLUT1_FWD & AGTATGTGGAGCAACTGTGT \\
\hline GLUT1_REV & TTGAAGTAGGTGAAGATGAAGAAC \\
\hline GPI_FWD & CCTGTCTACTAACACAACCAA \\
\hline GPI_REV & CACCCAATCCCAGAACTC \\
\hline LDHA_FWD & GGTTGAGAGTGCTTATGA \\
\hline LDHA_REV & AACACTAAGGAAGACATCA \\
\hline PGK1_FWD & GAAGCGGGTCGTTATGAG \\
\hline PGK1_REV & GCCTTAATCCTCTGGTTGTT \\
\hline PKM2_FWD & TCGGAGGTTTGATGAAAT \\
\hline PKM2_REV & TCTCCAGCATCTGAGTAG \\
\hline TPI_FWD & TATCATTTATGGAGGCTCTG \\
\hline TPI_REV & ATTGATGATGTCCACGAATT \\
\hline HK2_FWD & TGTGAGGTCCACTCCAGAT \\
\hline HK2_REV & GAGCCCATTGTCCGTTACTT \\
\hline TBP_FWD & TTCGGAGAGTTCTGGGATTGTA \\
\hline TBP_REV & TGGACTGTTCTTCACTCTTGGC \\
\hline
\end{tabular}


Table 2. Western Blotting and Immunohistochemistry Antibodies

\begin{tabular}{|c|c|}
\hline Target & Product \# \\
\hline P-S6K & $\# 9205$ \\
\hline S6K & $\# 2708$ \\
\hline P-ERK1/2 & $\# 4370$ \\
\hline ERK1/2 & $\# 9102$ \\
\hline B-Actin & $\#$ A1978 \\
\hline HK1 & $\# 2024$ \\
\hline HK2 & $\# 2867$ \\
\hline Ki67 & $\# 9449$ \\
\hline
\end{tabular}


A

Growth media discoloration after 48 hrs

Caki-1

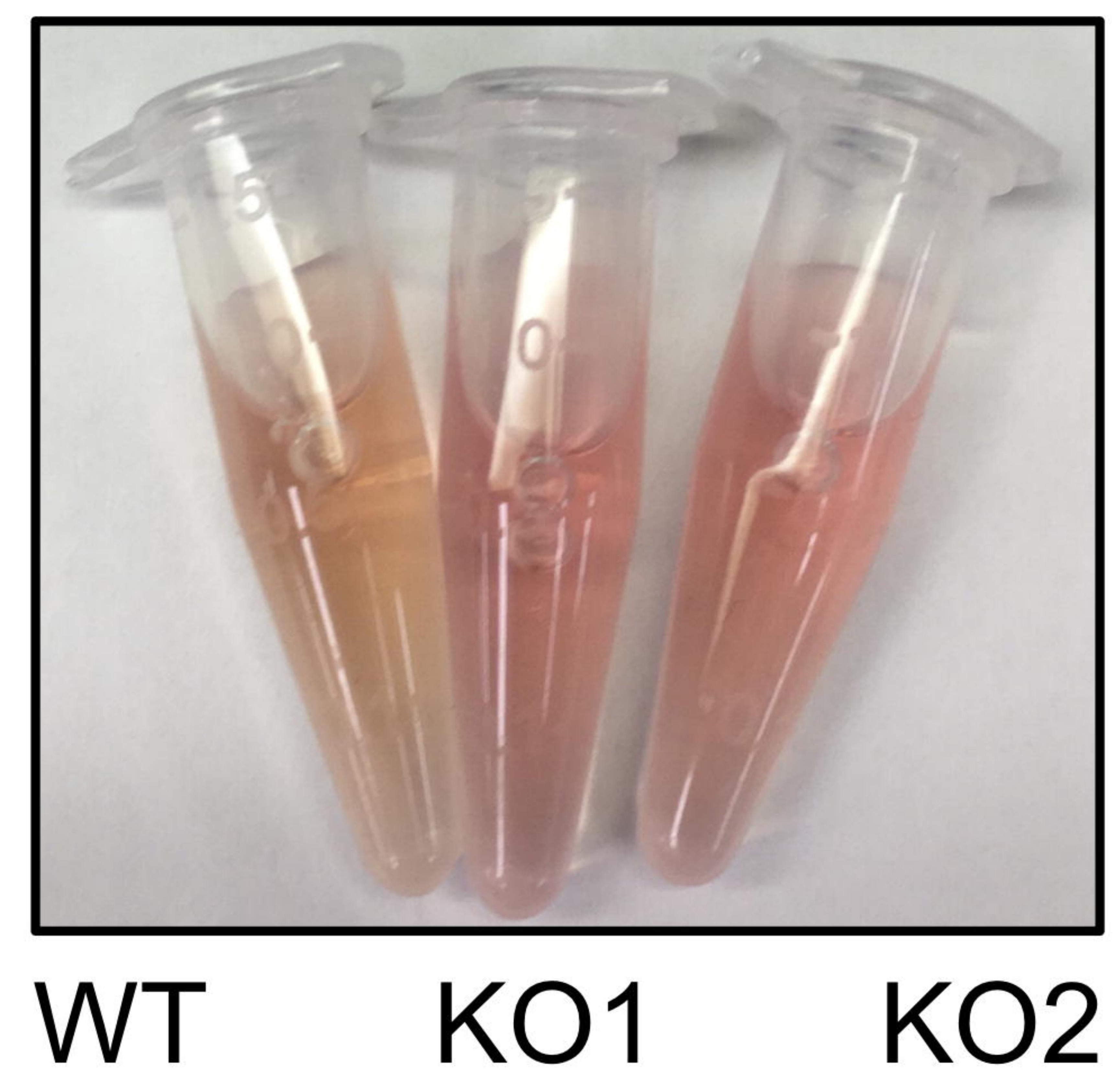

C

786-O

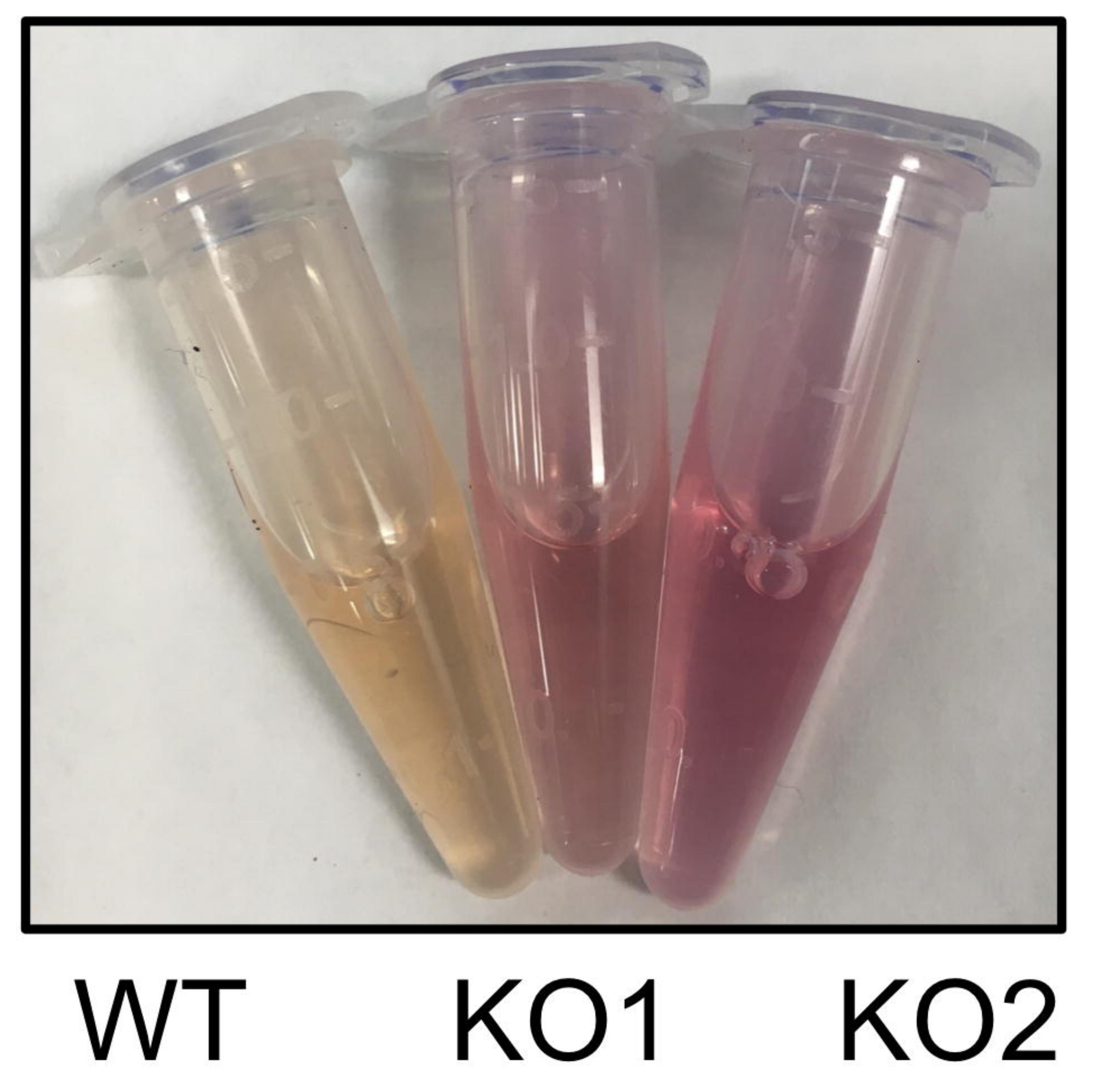

Glycolysis Brief Overview

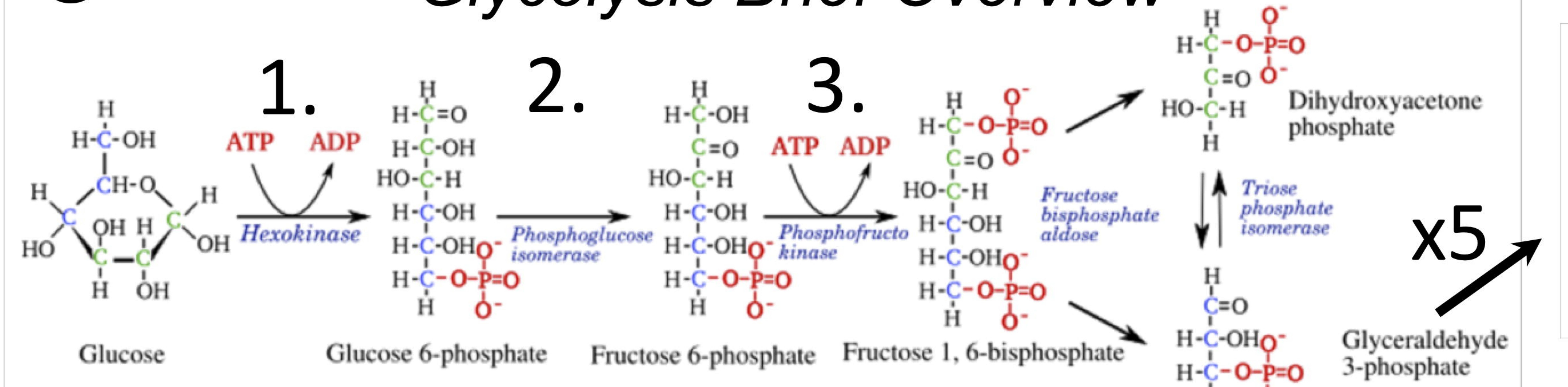

Glucose

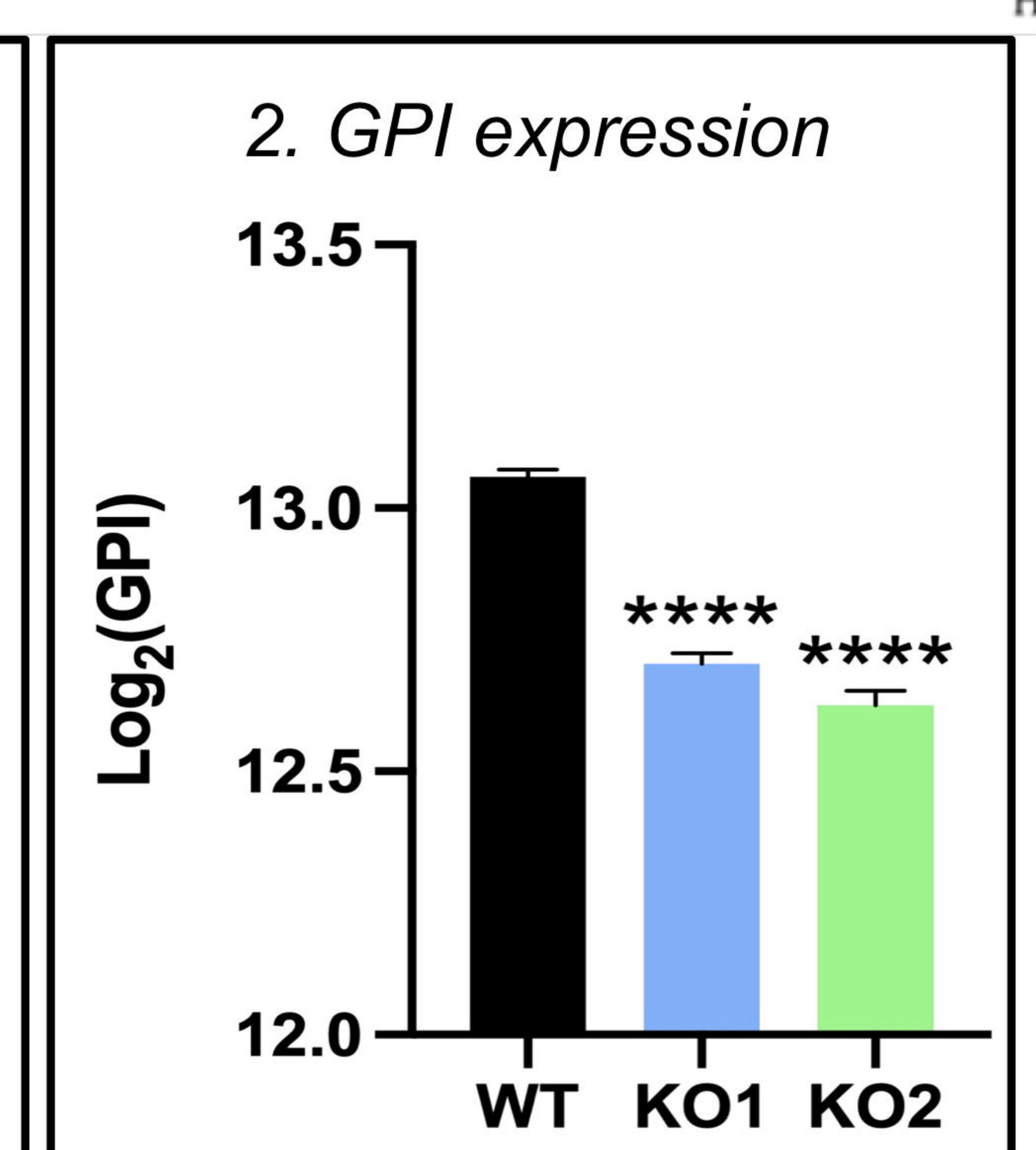

B

Gene Set Enrichment Analysis (GSEA) from MBOAT7 deficiency

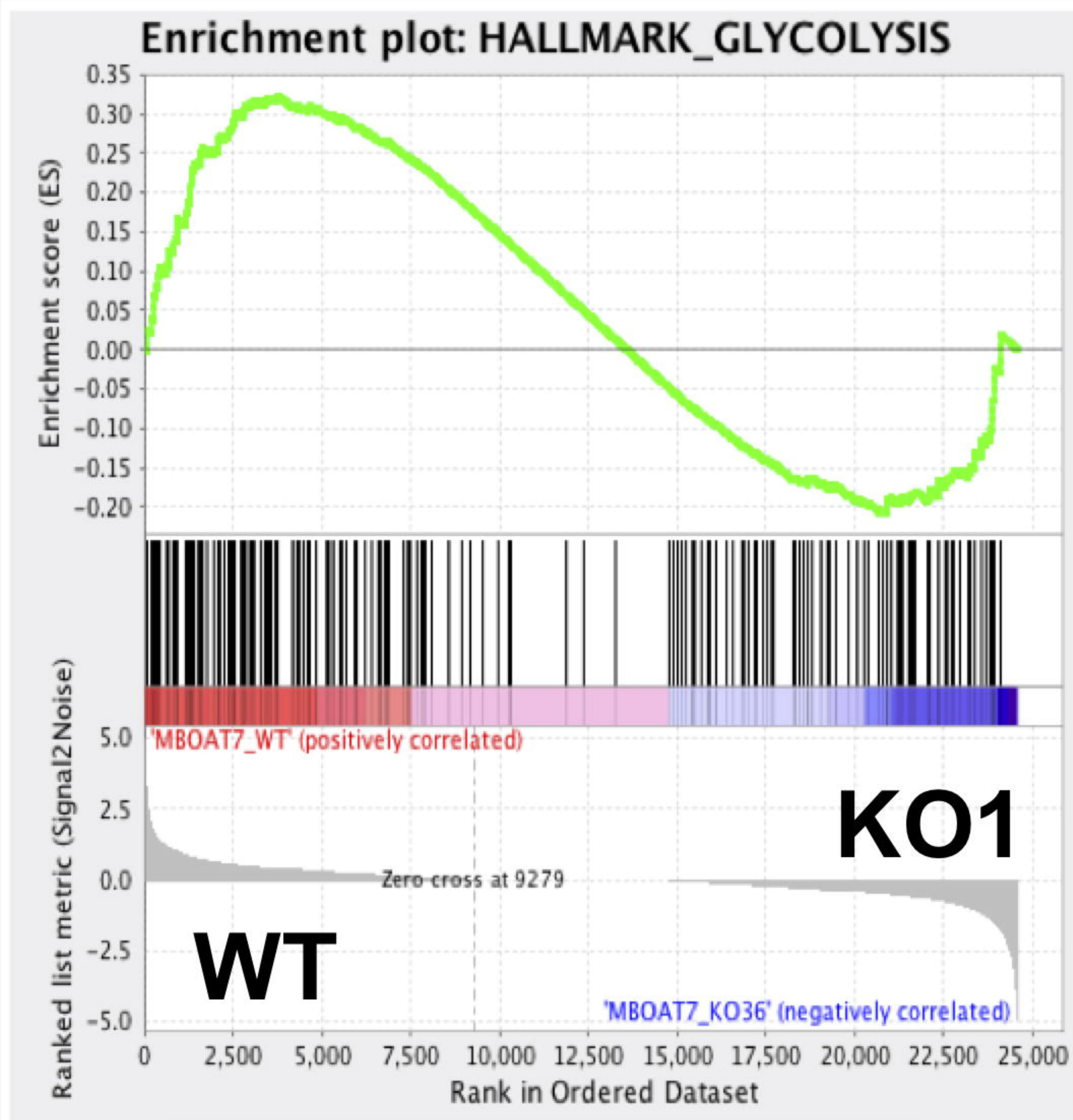

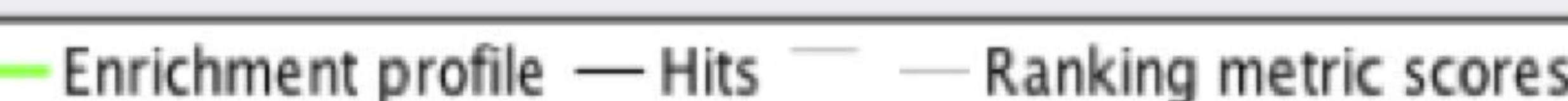

D

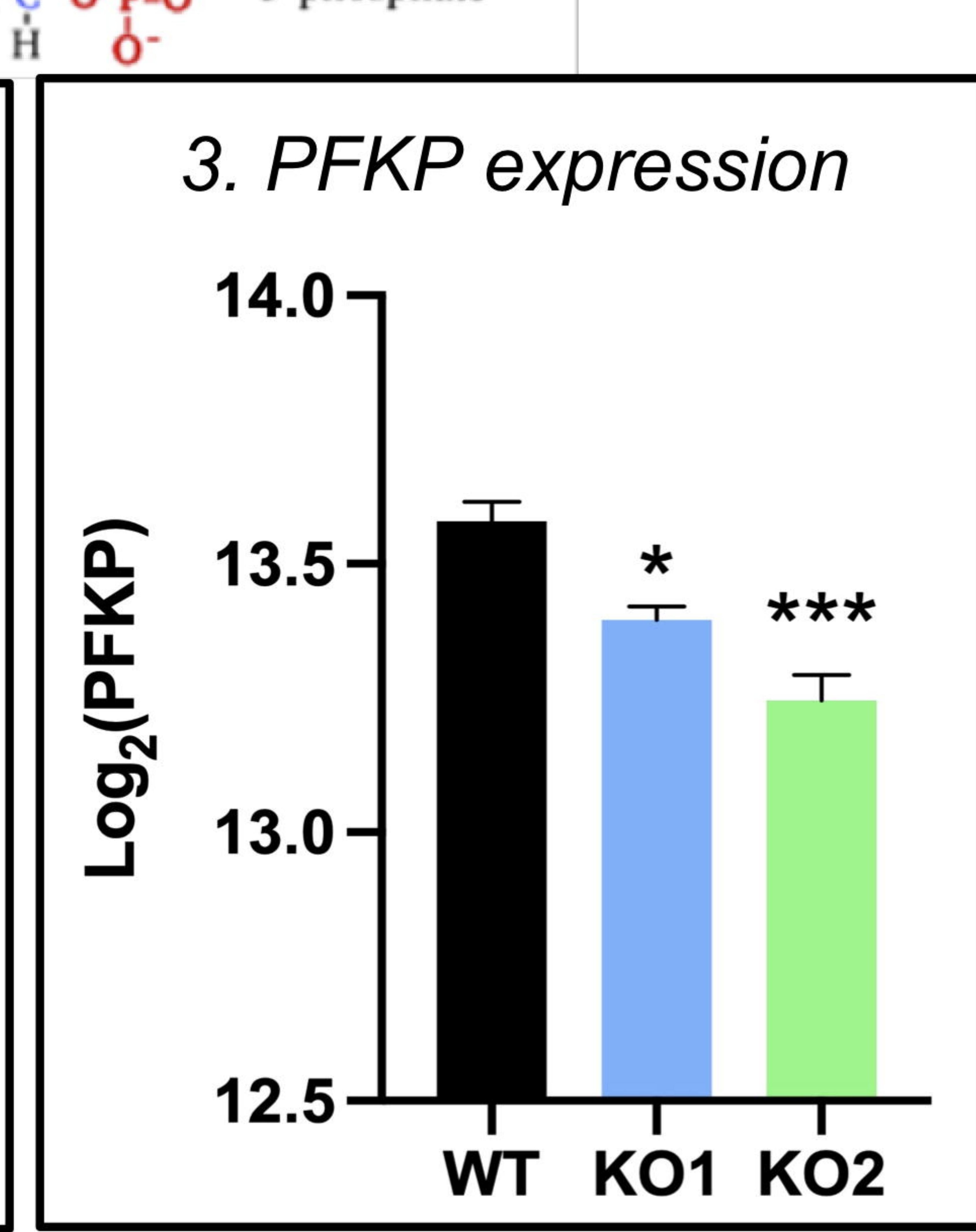

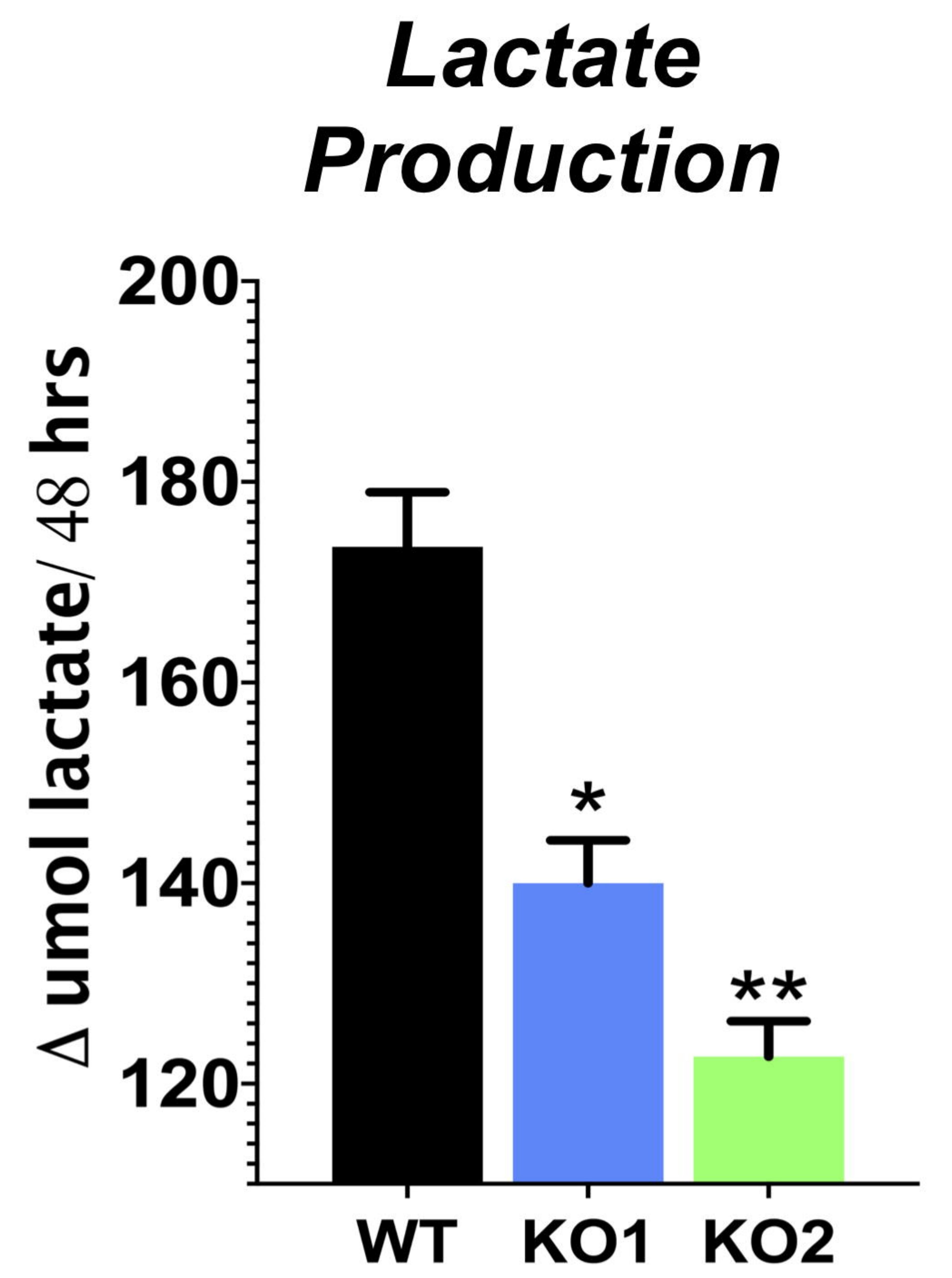

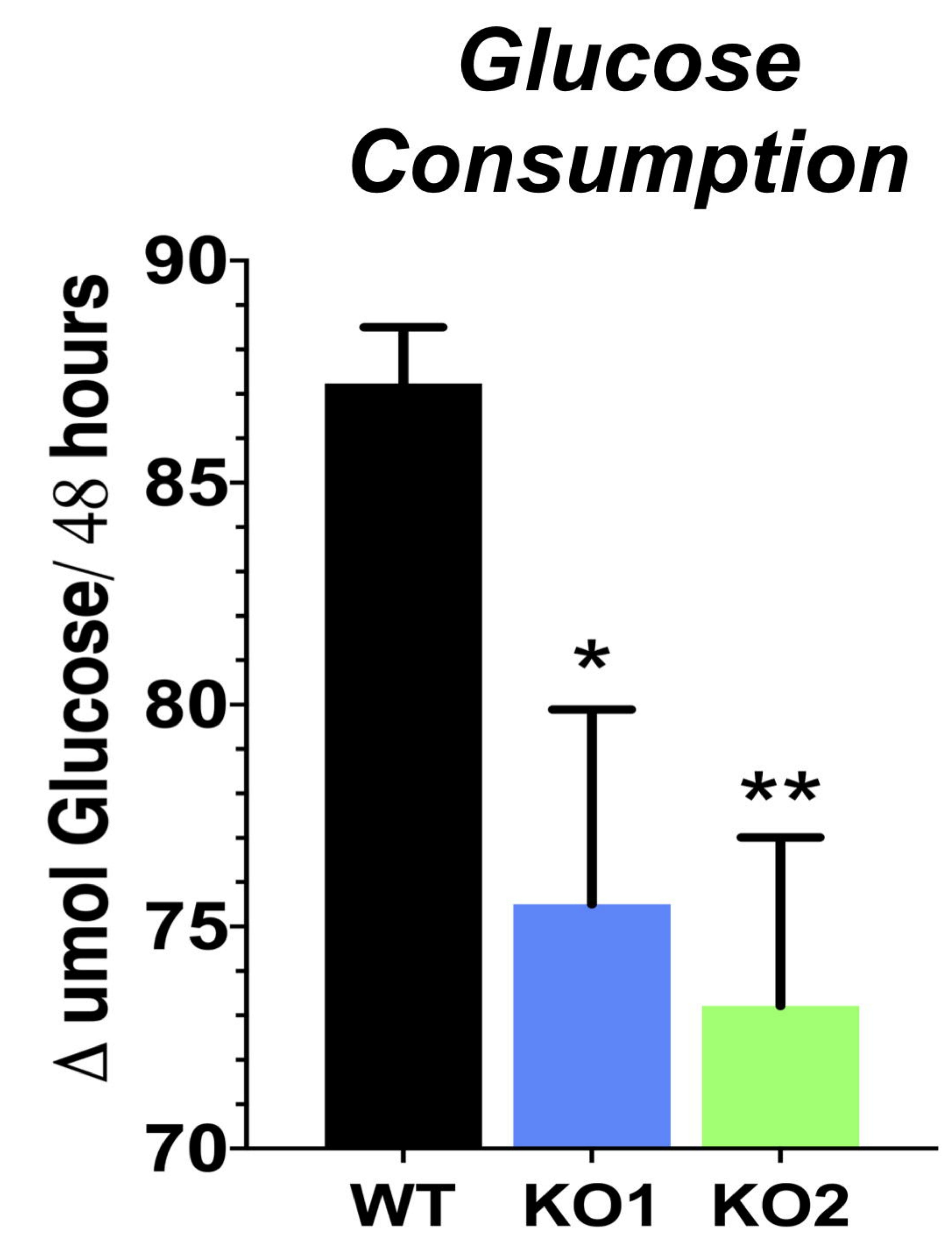

WT KO1 KO2
Enrichment plot: HALLMARK_GLYCOLYSIS

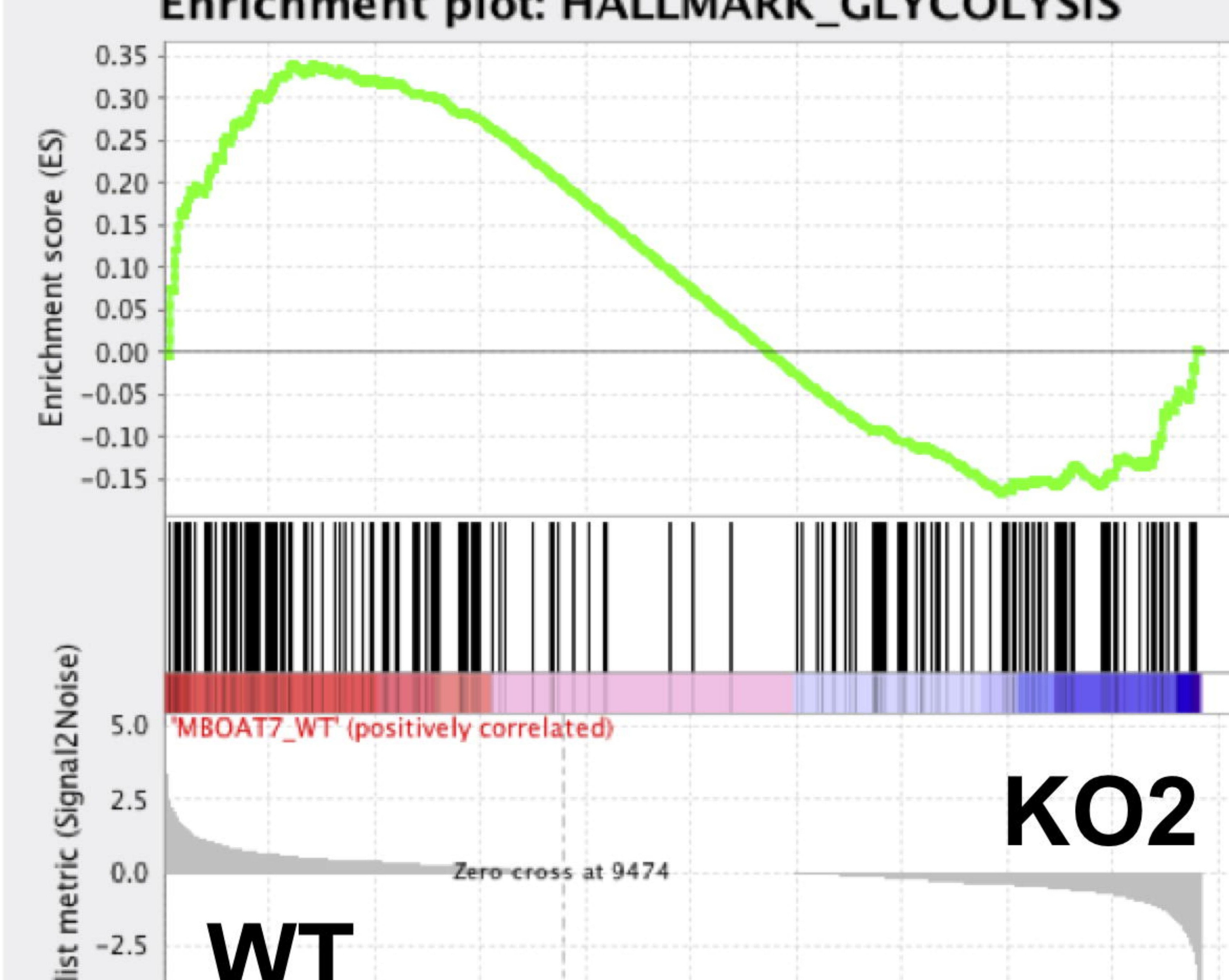

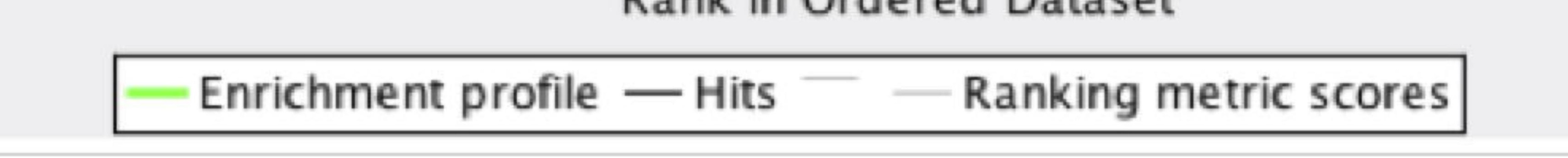


A
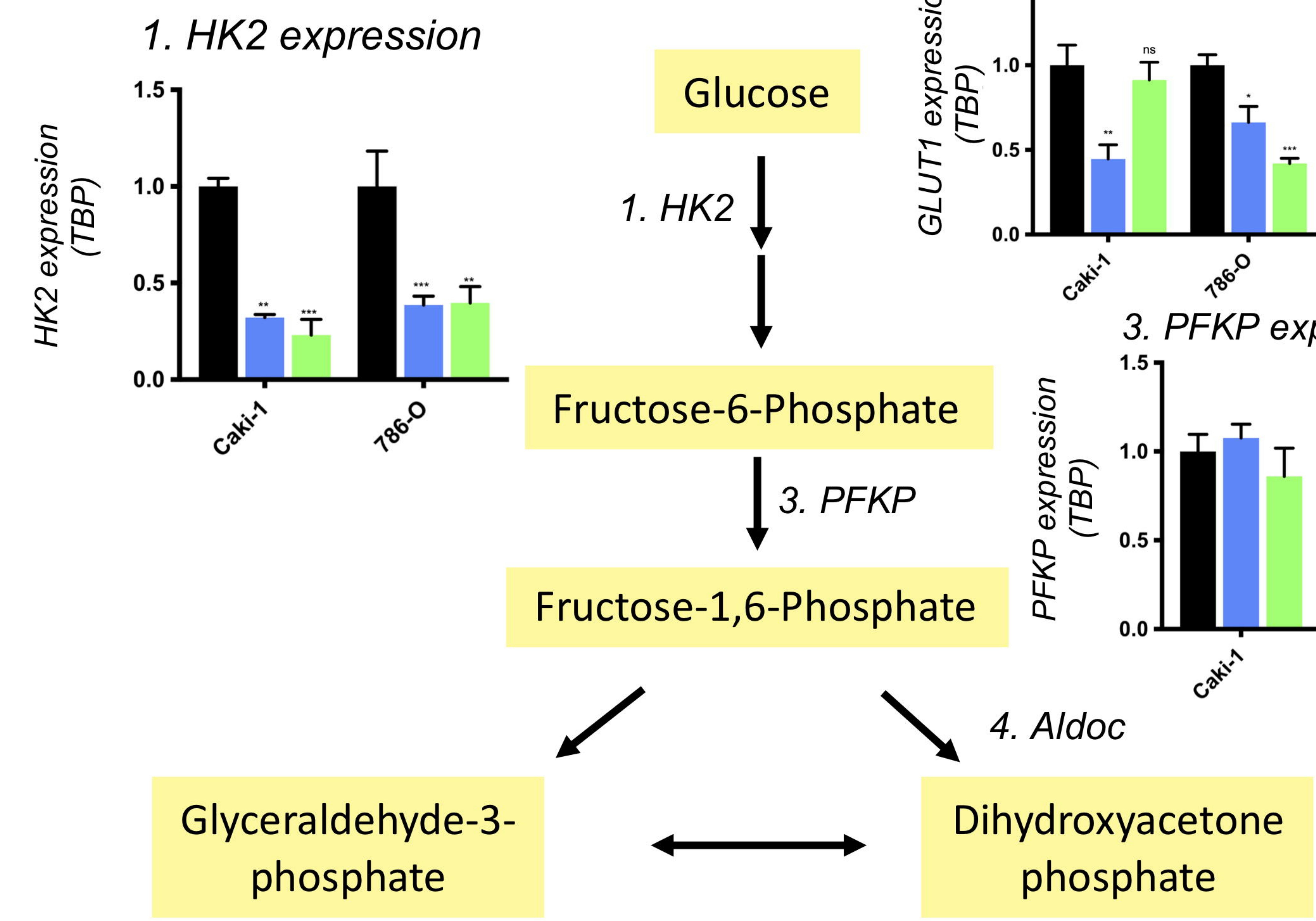

Glyceraldehyde-3 phosphate

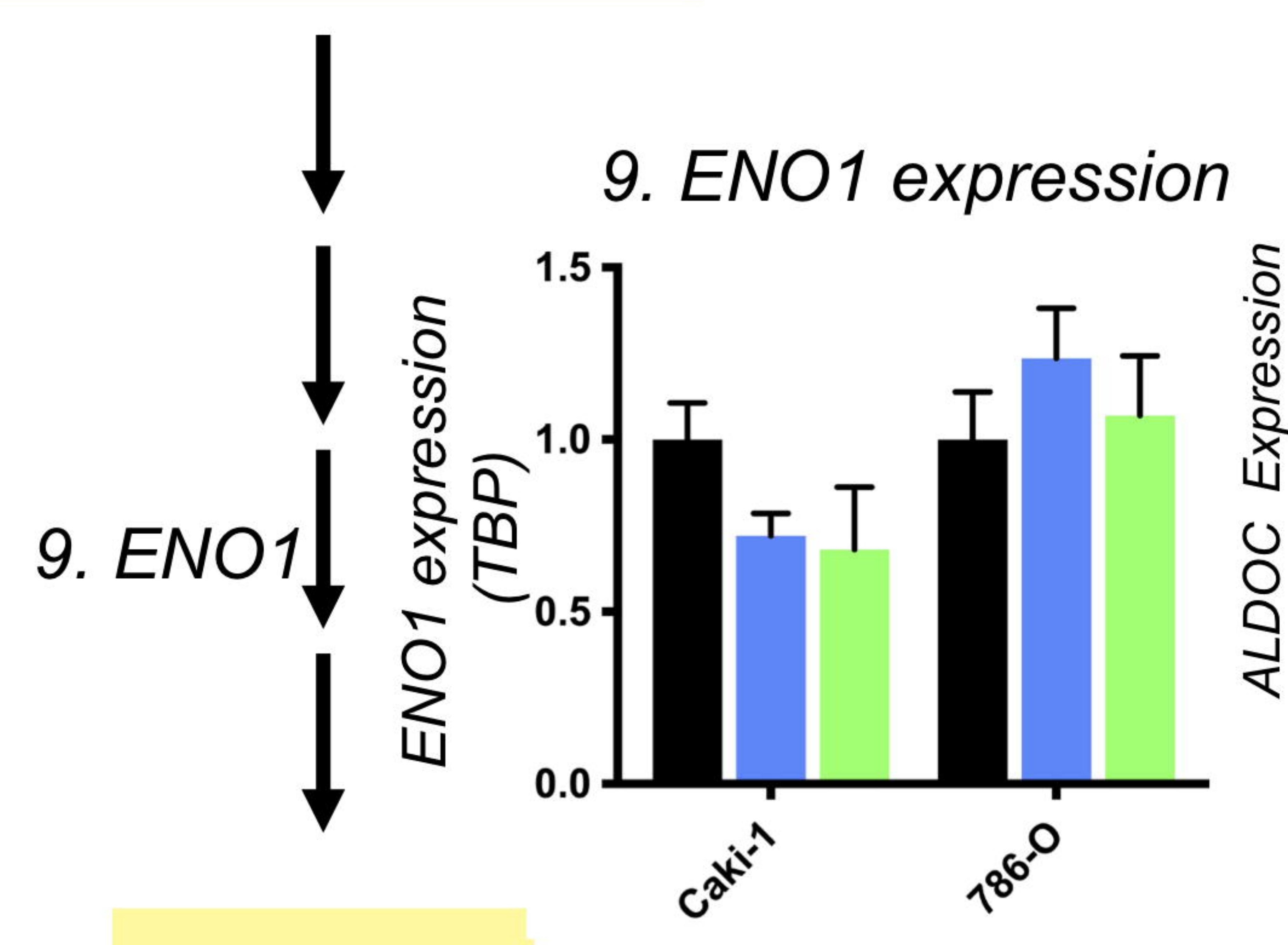

Pyruvate
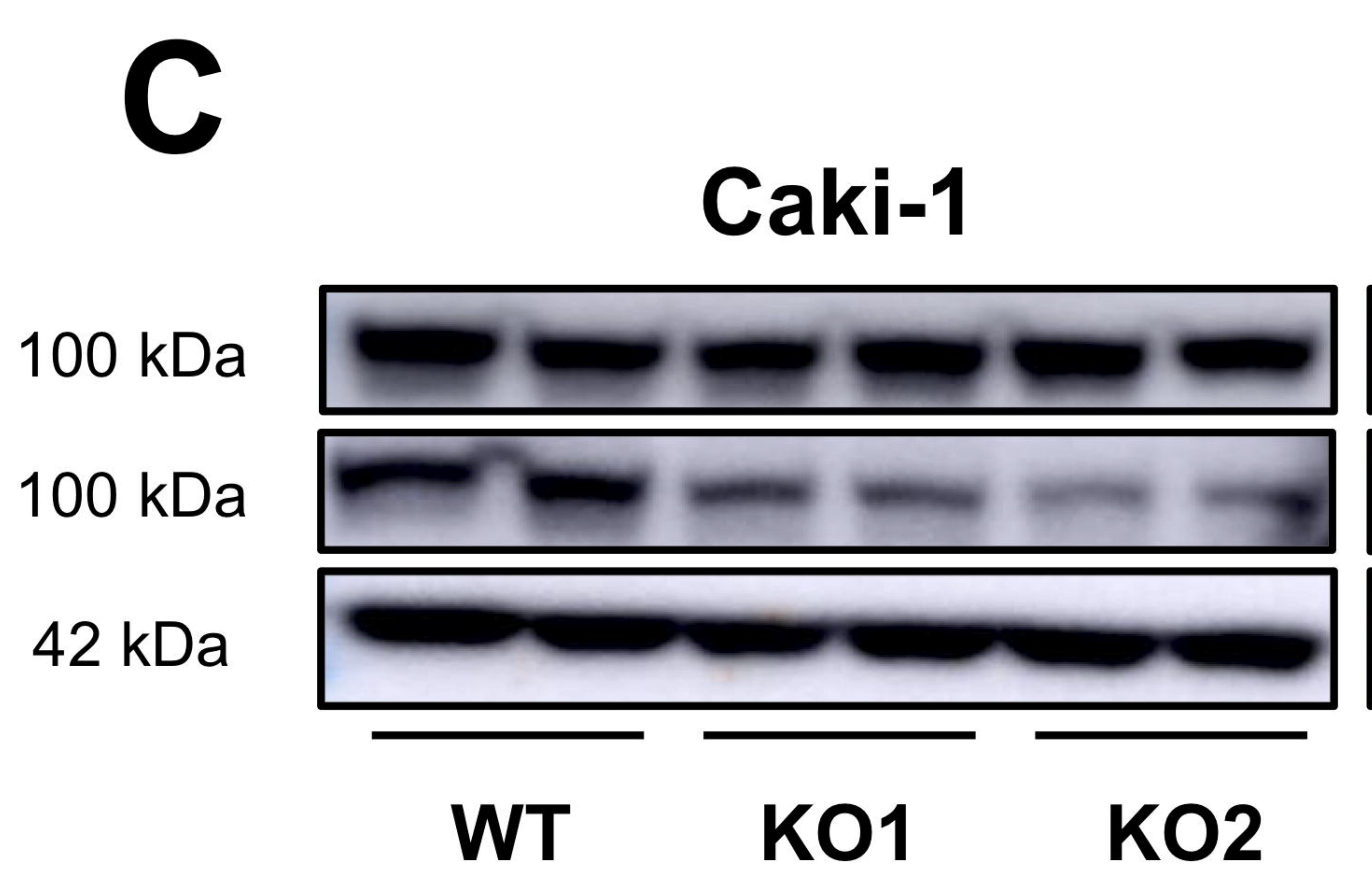

B

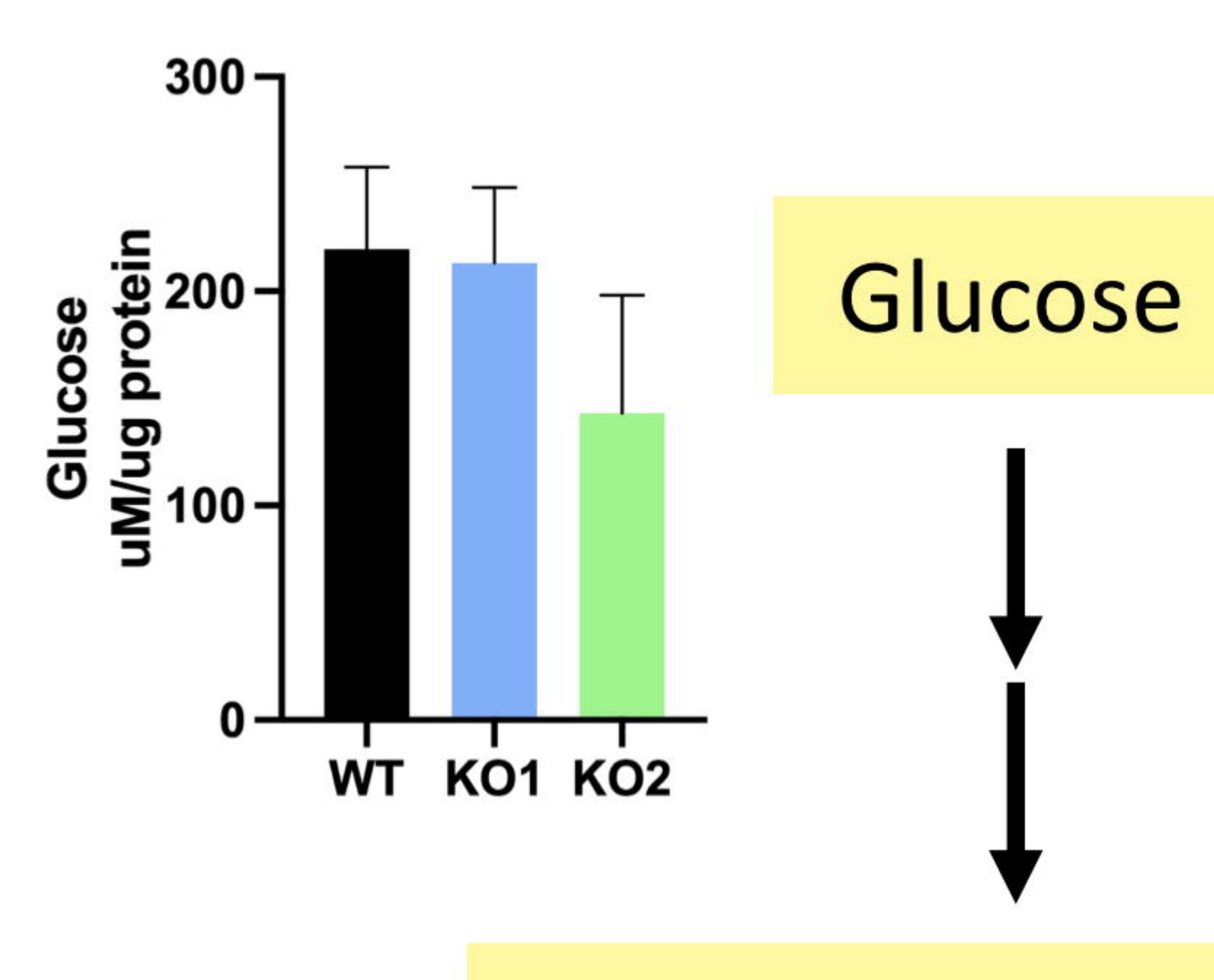

Fructose-6-Phosphate

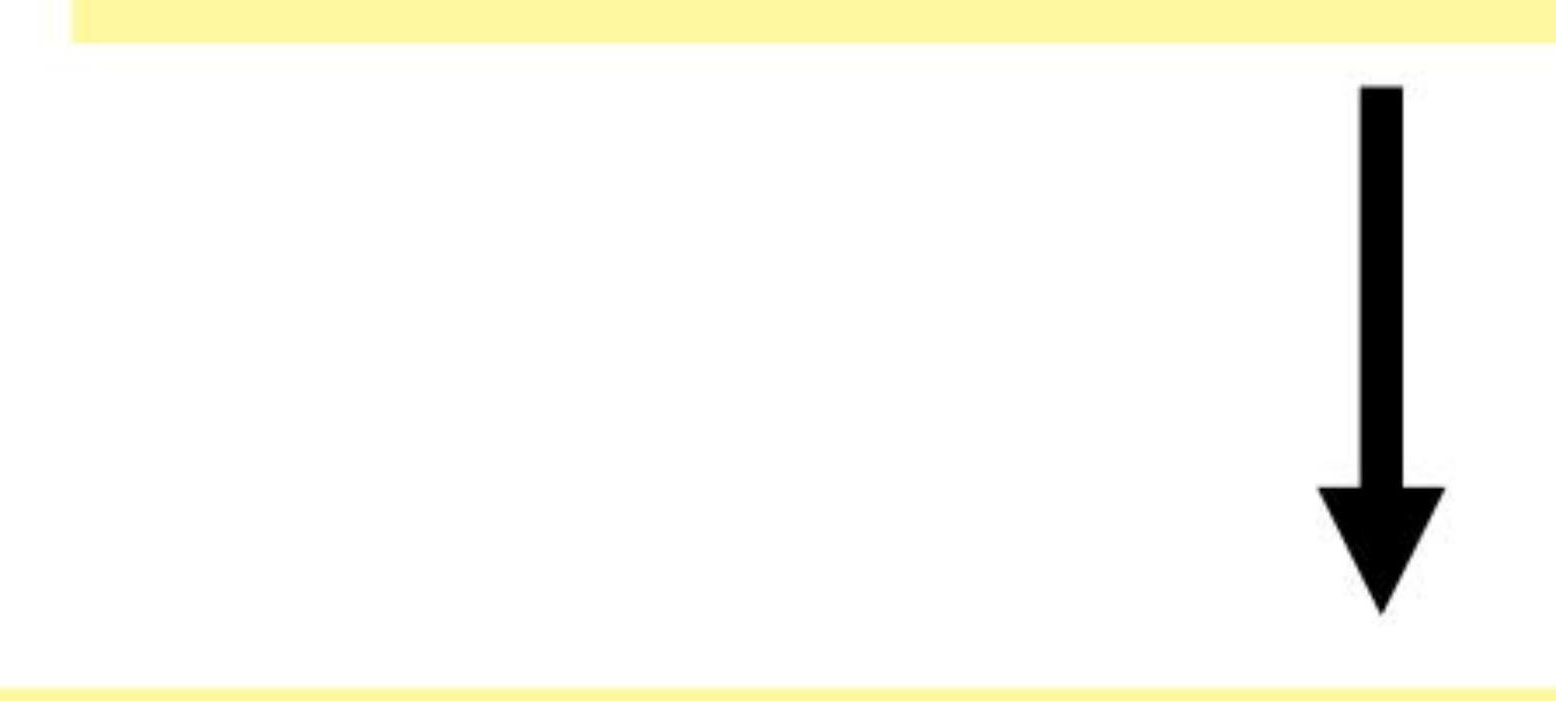

Fructose-1,6-Phosphate

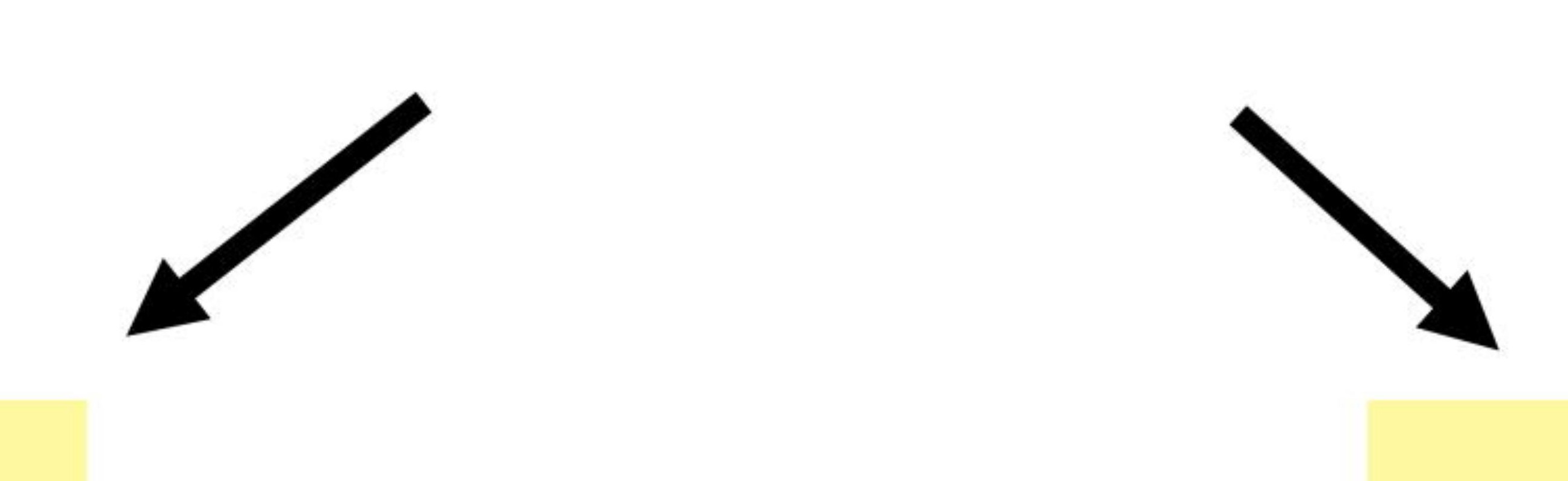

Glyceraldehyde-3phosphate

Dihydroxyacetone phosphate

4. ALDOC expression

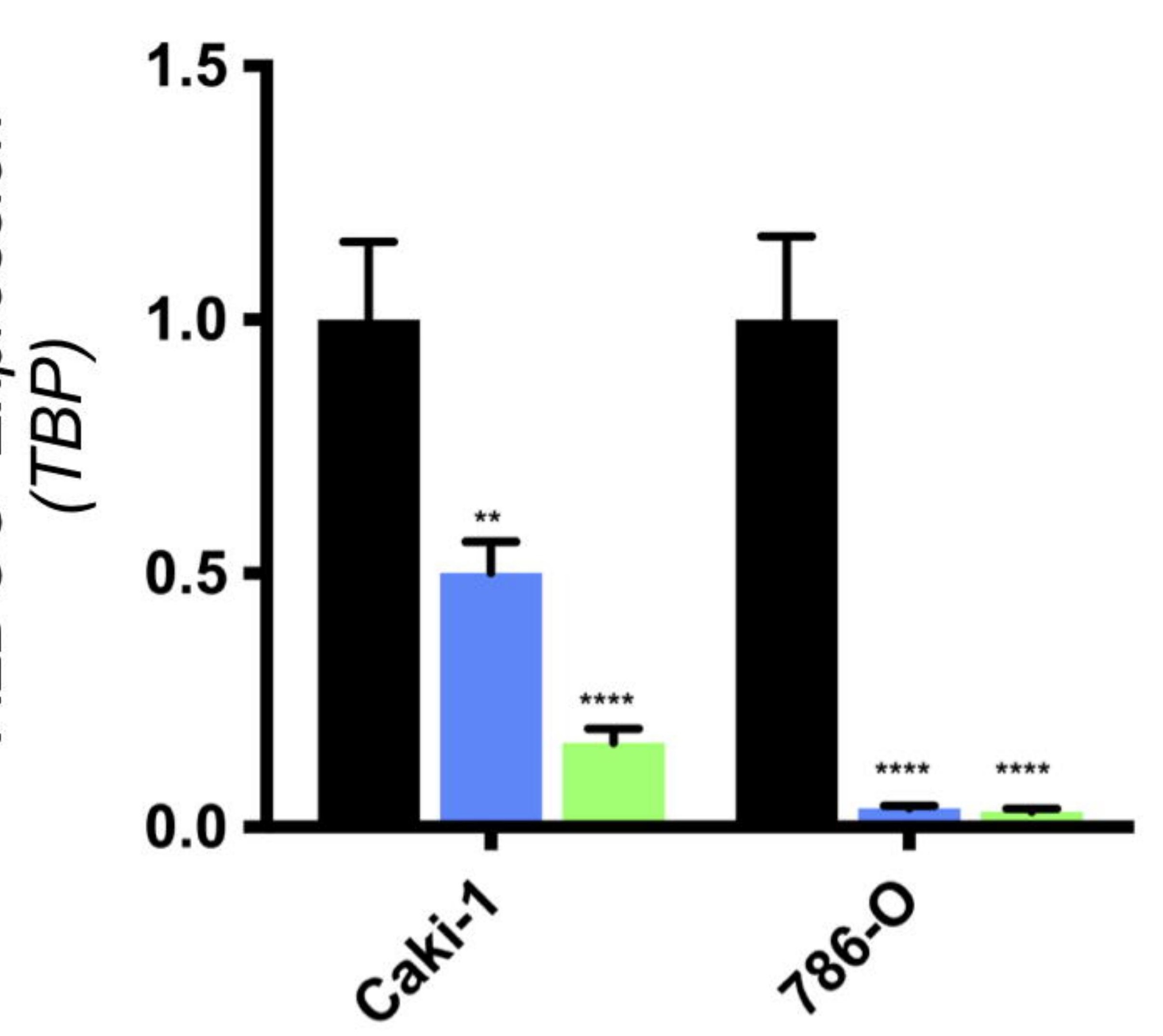

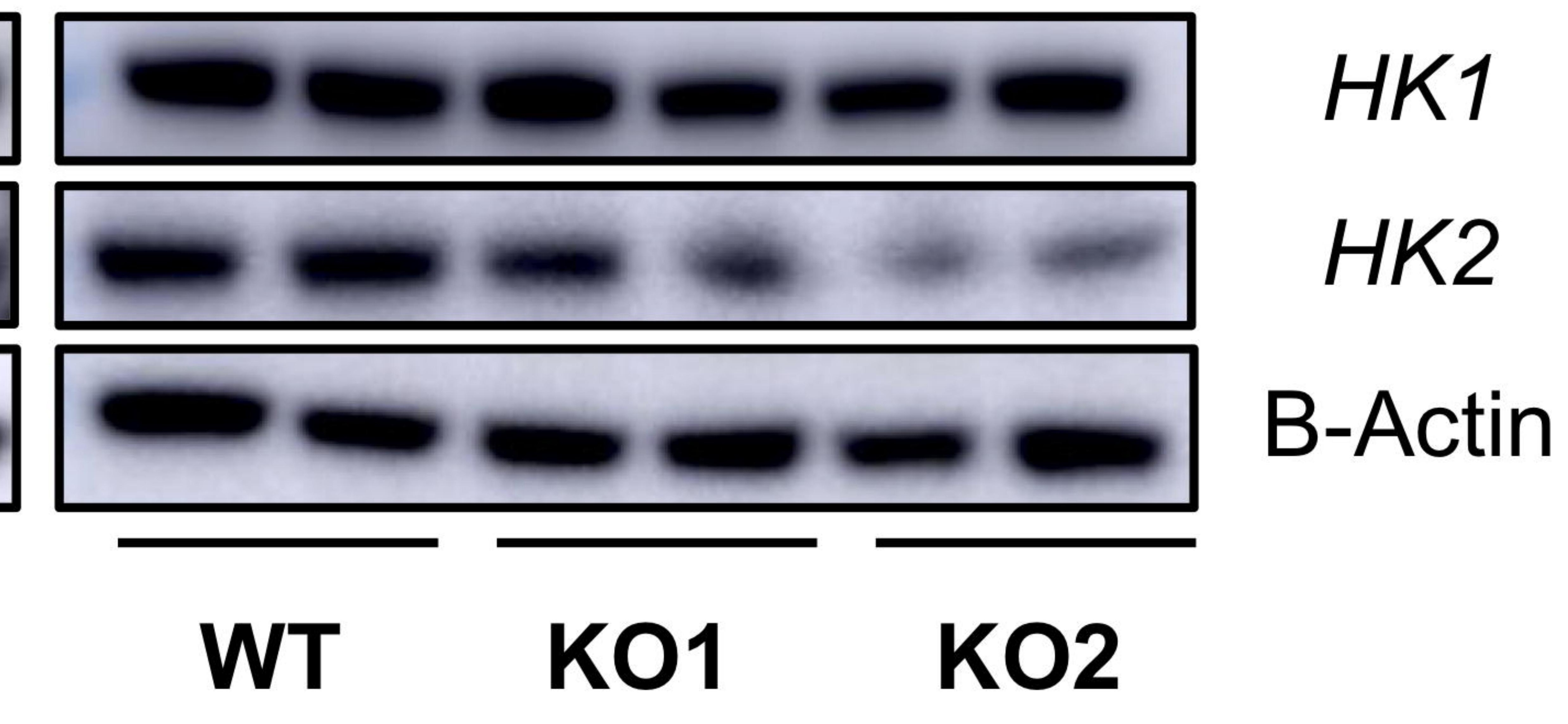



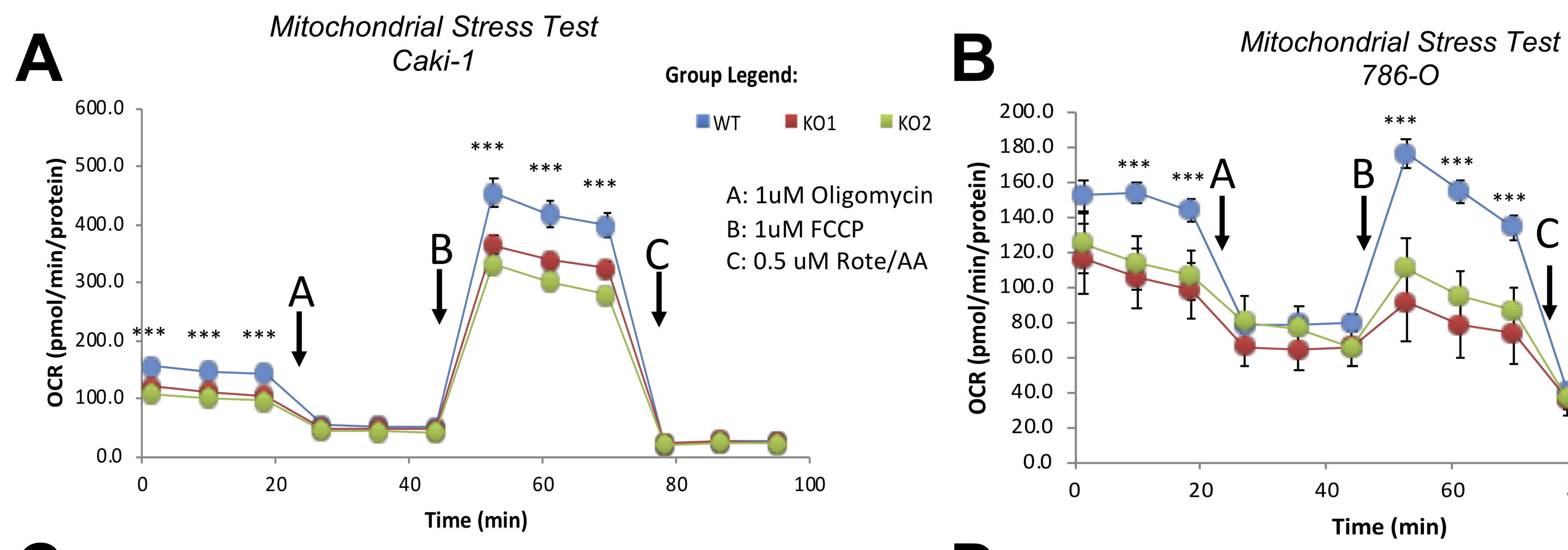

\section{C}

\section{Caki-1}
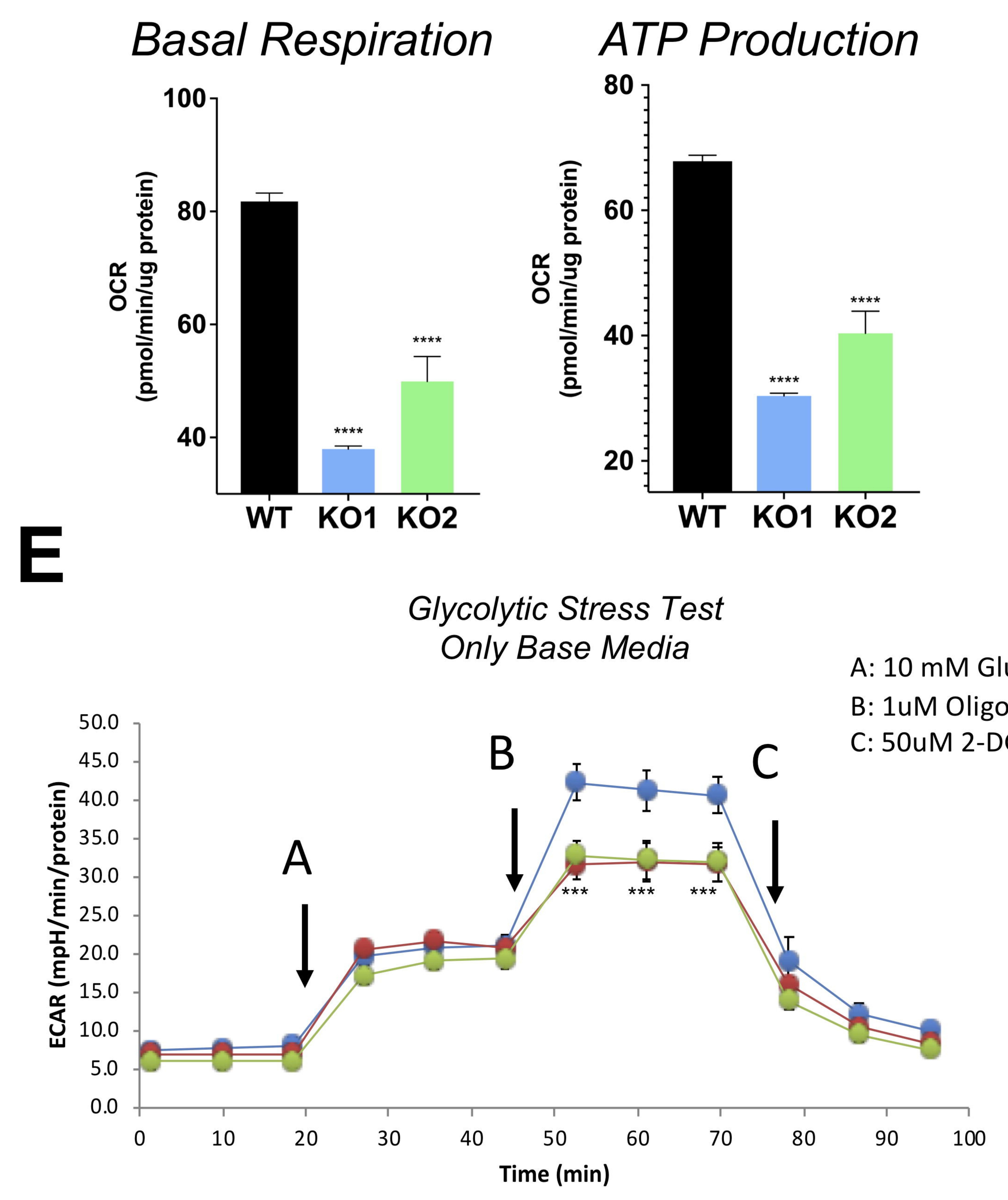

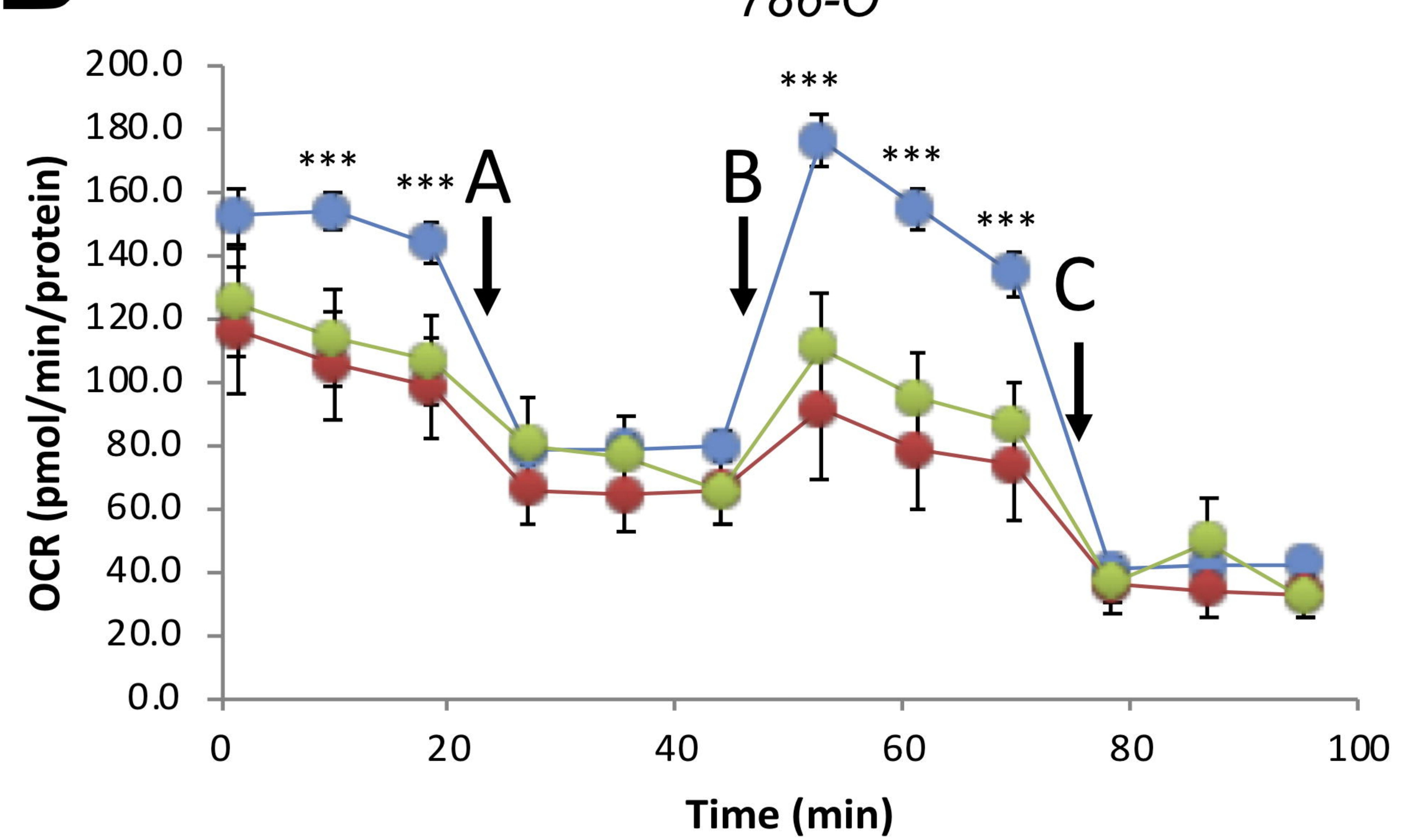

D

786-O

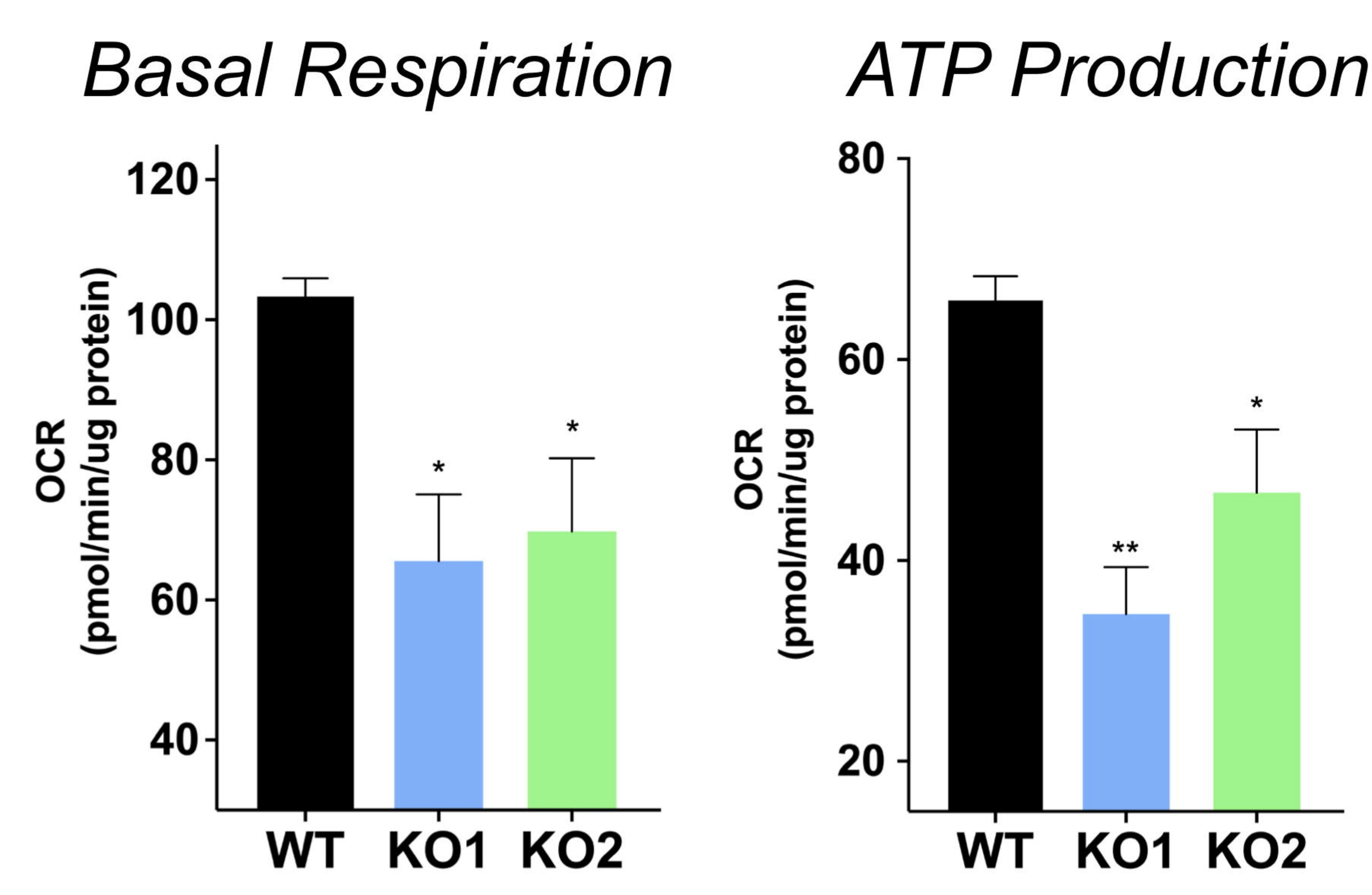

F

Glycolytic Stress Test Base Media + 1mM Pyruvate

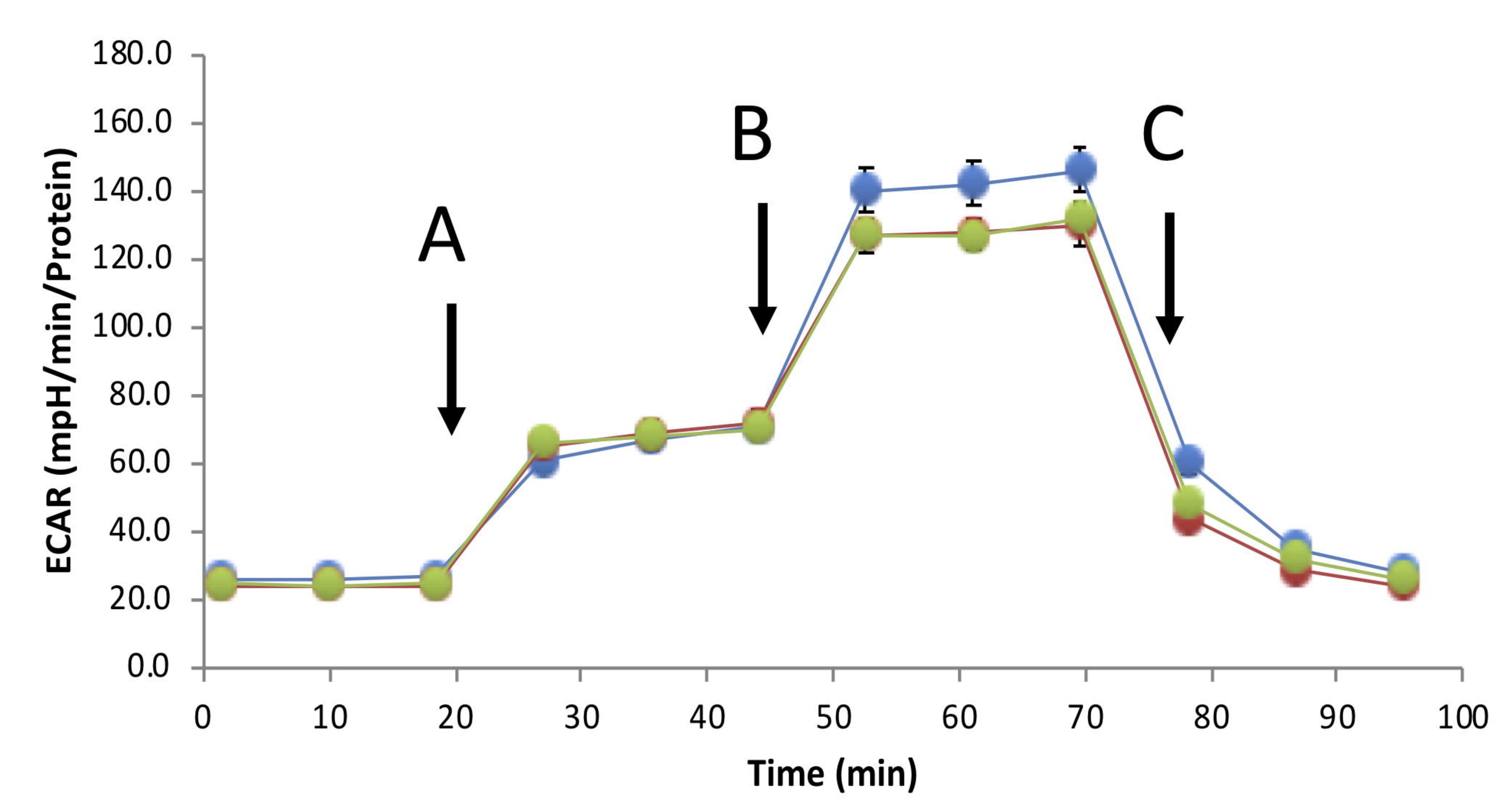




\section{A MBOAT7 KO increased survival in 786-O xenograft}
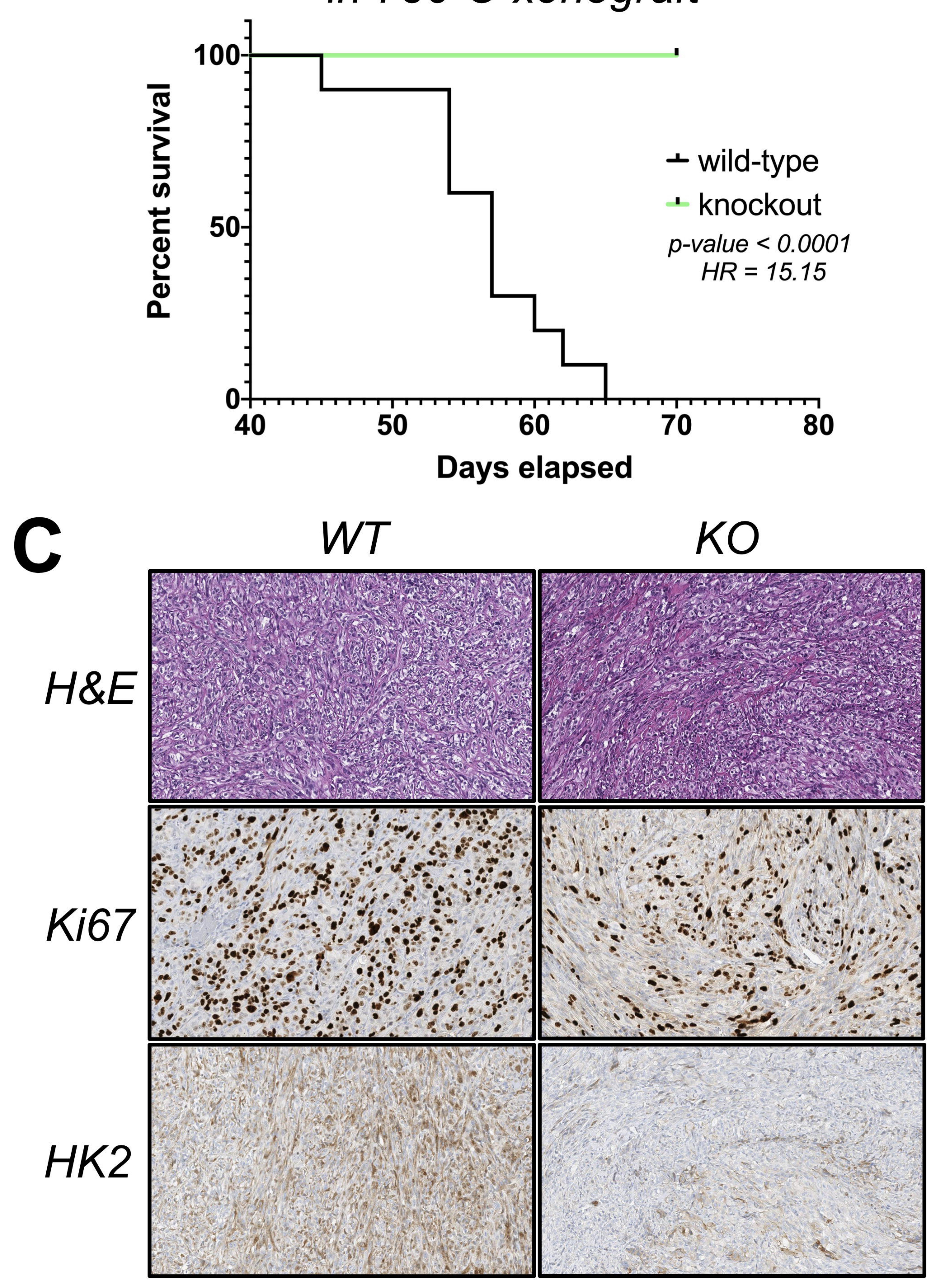

E

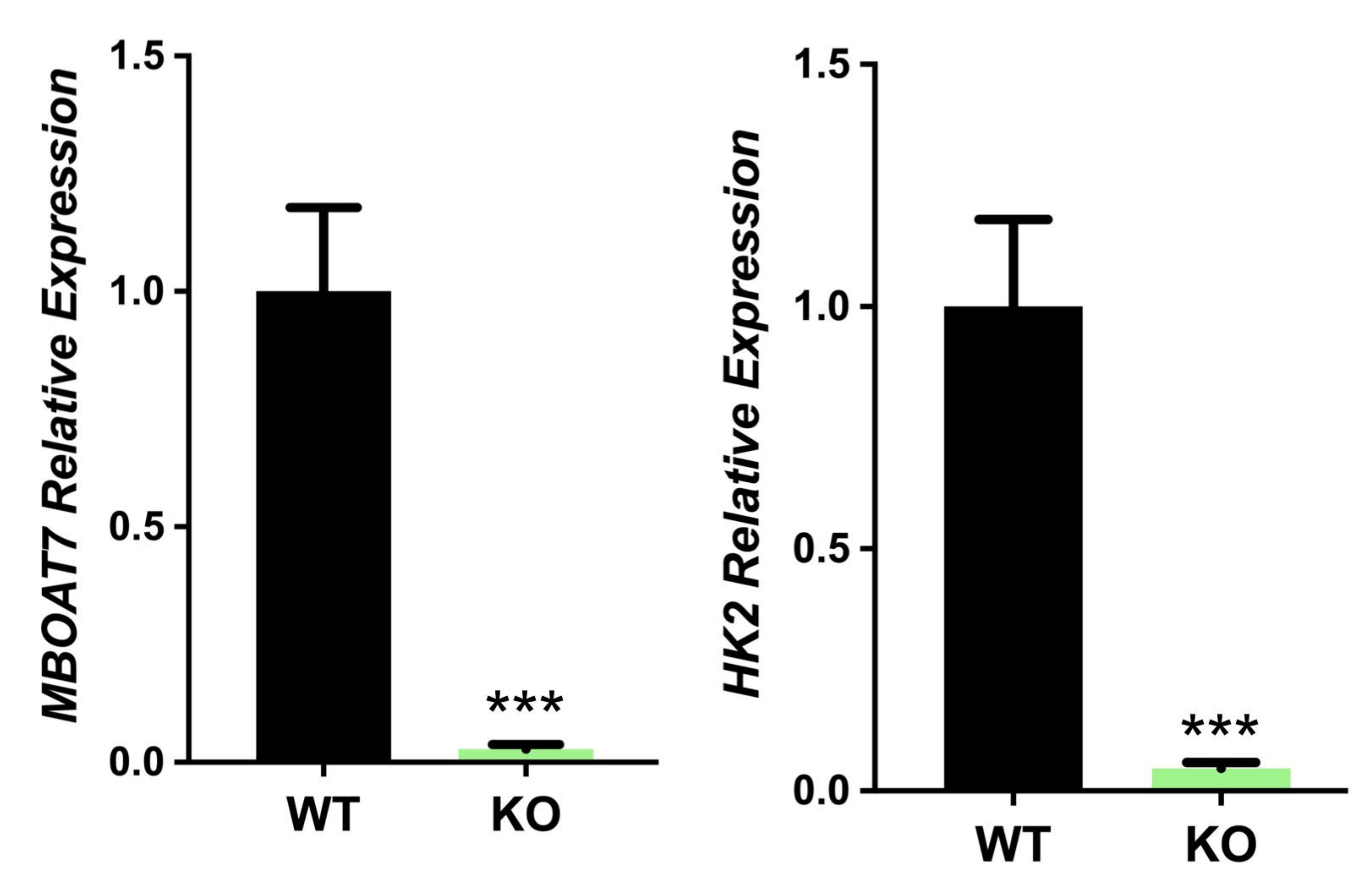

B

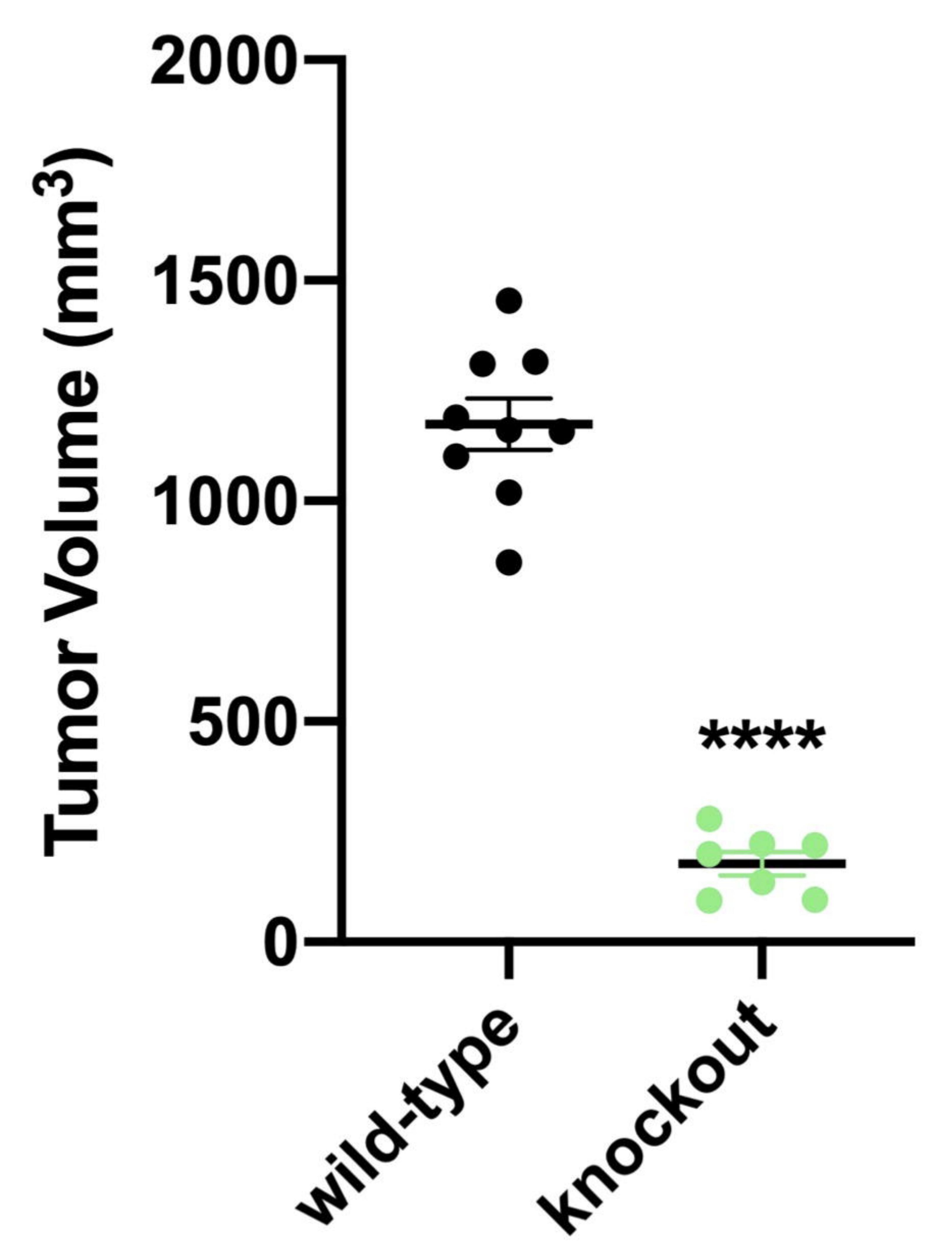

D

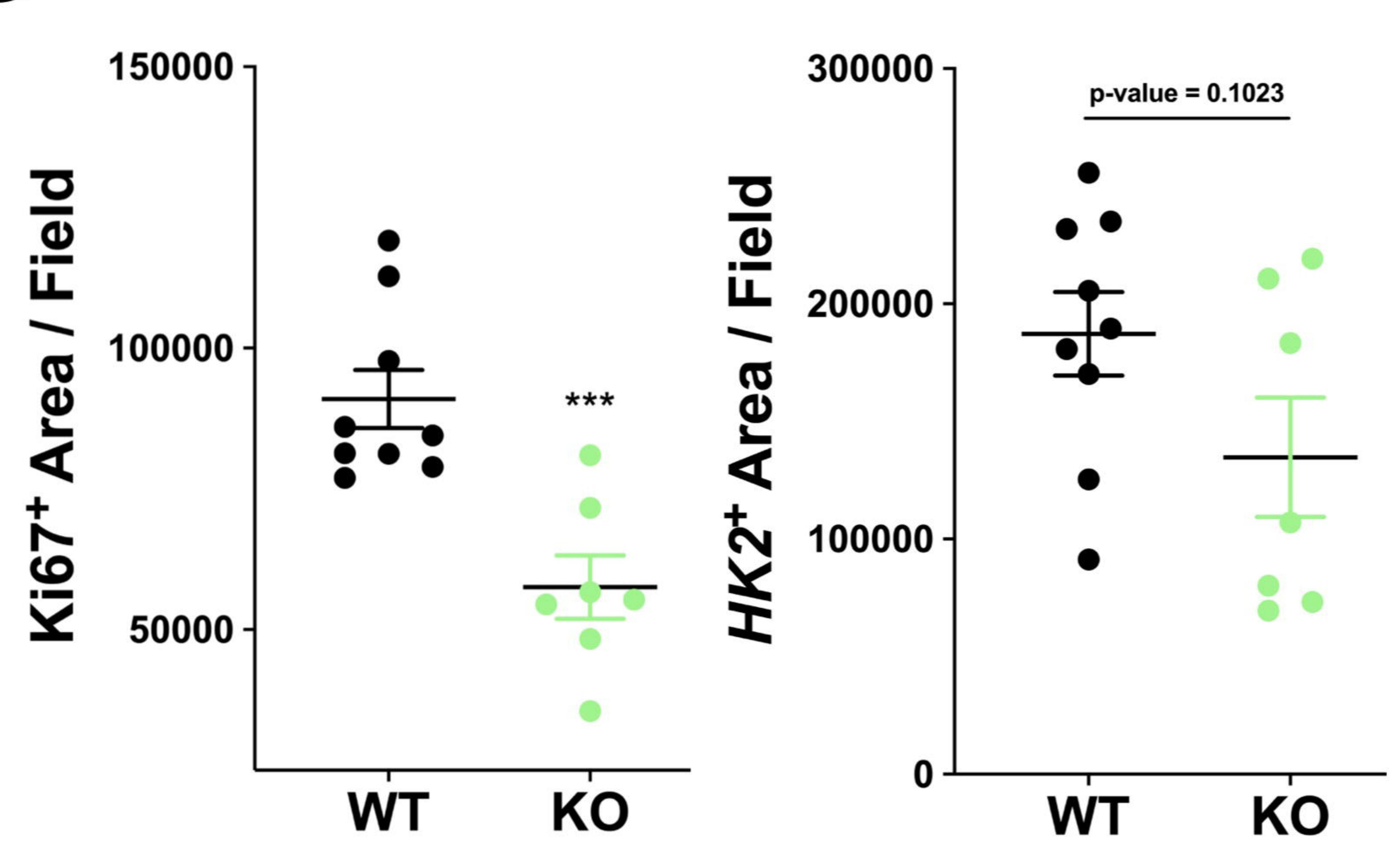

F Tumors: $W T \quad K O$

S6K

P-ERK $\approx \equiv=\equiv=$

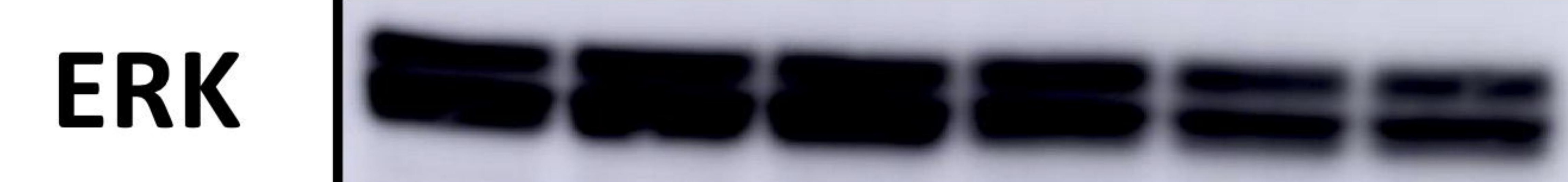

HK2 $-\infty--\cdots$

HK1 $-\infty$

B-Actin 